Supporting Information For

\title{
Synthesis and Fungicidal Evaluation of Novel Chalcone-based
}

\section{Strobilurins Analogues}

\author{
Pei-Liang Zhao, ${ }^{\mathrm{a}}$ Chang-Ling Liu, ${ }^{\mathrm{b}}$ Wei Huang, ${ }^{\mathrm{a}}$ Ya-Zhou Wang, ${ }^{\mathrm{a}}$ Guang-Fu Yang ${ }^{\mathrm{a}, *}$ \\ ${ }^{a}$ Key Laboratory of Pesticide \& Chemical Biology, Ministry of Education, College of Chemistry, Central \\ China Normal University, Wuhan 430079, P.R.China \\ ${ }^{\mathrm{b}}$ Shenyang Research Institute of Chemical Industry, Agrochemical Discovery Department, Shenyang 110021, P. \\ R. China
}

"To whom correspondence should be addressed. Fax: 86-27-67867141, E-mail: gfyang@ mail.ccnu.edu.cn

Preparation of methyl 2-o-tolylacetate (4). To a $250 \mathrm{~mL}$ round-bottom flask, $30.0 \mathrm{~g}(0.2 \mathrm{~mol})$ of 2-o-tolylacetic acid, $5 \mathrm{~mL}$ of concentrated sulfuric acid and $100 \mathrm{~mL}$ of methanol were added gradually. The resulting mixture was stirred with heating for $8 \mathrm{~h}$ and was then concentrated on a rotary evaporator. The crude product obtained was poured into $200 \mathrm{ml}$ of water and extracted with ethyl acetate $(100 \mathrm{~mL} \times 3)$. The combined ethyl acetate extracts were washed with water $(100$ $\mathrm{mL} \times 2$ ), dried with anhydrous magnesium sulfate, filtered off by suction and the solvent was evaporated to give crude, light yellow liquids $\mathbf{4}$ which could be purified by column chromatography using a mixture of petroleum ether and ethyl acetate $(6: 1)$ as an eluent. Yield 94\%. ${ }^{1} \mathrm{H}$ NMR $\left(\mathrm{CDCl}_{3}\right): \delta 2.34\left(\mathrm{~s}, 3 \mathrm{H}, \mathrm{CH}_{3}\right), 3.58\left(\mathrm{~s}, 2 \mathrm{H}, \mathrm{CH}_{2}\right), 3.63\left(\mathrm{~s}, 3 \mathrm{H}, \mathrm{OCH}_{3}\right), 7.01-7.29(\mathrm{~m}$, 4H, ArH). EI MS: $m / z$ (\%) $164\left(\mathrm{M}^{+}, 47\right), 133$ (100), 31 (75).

Preparation of methyl 3-hydroxy-2-o-tolylacrylate (5). To a $500 \mathrm{~mL}$ round-bottom flask, 24.6 $\mathrm{g}(0.15 \mathrm{~mol})$ of methyl 2-o-tolylacetate, $10.8 \mathrm{~g}(0.45 \mathrm{~mol})$ of $60 \%$ sodium hydride were added $300 \mathrm{~mL}$ of anhydrous $\mathrm{N}, \mathrm{N}$-dimethylformamide (DMF) at $0 \square$, and $27.0 \mathrm{~g}(0.45 \mathrm{~mol})$ of methylformate was added dropwise thereto over a period of $1 \mathrm{~h}$ while cooling and stirring. The resulting solution was stirred for $12 \mathrm{~h}$ at room temp,erature and extracted with water $(300 \mathrm{~mL} \times 3)$. The combined aqueous layer was acidified with concentrated hydrochloric acid and then extracted with ethyl acetate $(100 \mathrm{~mL} \times 3)$. the combined organic layers were dried over anhydrous magnesium sulfate, filtered, and the filtrate was evaporated to afford a light yellow liquid, which was chromatographed on silica with petroleum ether and ethyl acetate $(12: 1)$ as an eluent to give colorless liquid 5. Yield 88\%. ${ }^{1} \mathrm{H}$ NMR $\left(\mathrm{CDCl}_{3}\right): \delta 2.34\left(\mathrm{~s}, 3 \mathrm{H}, \mathrm{CH}_{3}\right), 3.73\left(\mathrm{~s}, 3 \mathrm{H}, \mathrm{OCH}_{3}\right)$, 7.05-7.46 (m, 5H, ArH), 11.92 (d, 1H, OH). EI MS: $m / z(\%) 192\left(\mathrm{M}^{+}, 27\right), 160(54), 132$ (46), 84 (100).

Preparation of (E)-methyl 3-methoxy-2-o-tolylacrylate (6). To $4.4 \mathrm{~g}(0.11 \mathrm{~mol})$ of $60 \%$ sodium hydride in $80 \mathrm{~mL}$ of anhydrous dimethyl formamide (DMF) was added to $19.2 \mathrm{~g}(0.1 \mathrm{~mol})$ of methyl 3-hydroxy-2-o-tolylacrylate 3 at $0 \square$. The resulting solution was stirred for $0.5 \mathrm{~h}$ at room temp,erature, and then, $15.1 \mathrm{~g}(0.12 \mathrm{~mol})$ of dimethyl sulfate was added. After $12 \mathrm{~h}$ at room 
temp,erature, the mixture was extracted with ethyl acetate, and the combined organic layers were washed with water $(50 \mathrm{~mL} \times 3)$, dried over anhydrous magnesium sulfate, filtered, and concentrated. The residue was subjected to column chromatography using a mixture of petroleum ether and ethyl acetate (20:1) as an eluent to give colorless liquid 6. Yield 77\%. ${ }^{1} \mathrm{H} \mathrm{NMR}\left(\mathrm{CDCl}_{3}\right)$ : $\delta 2.23\left(\mathrm{~s}, 3 \mathrm{H}, \mathrm{CH}_{3}\right), 3.68\left(\mathrm{~s}, 3 \mathrm{H}, \mathrm{COOCH}_{3}\right), 3.79\left(\mathrm{~s}, 3 \mathrm{H}, \mathrm{OCH}_{3}\right), 6.97-7.36(\mathrm{~m}, 4 \mathrm{H}, \mathrm{ArH}), 7.51$ (s, $1 \mathrm{H}, \mathrm{C}=\mathrm{CH})$. EI MS: $m / z(\%) 206\left(\mathrm{M}^{+}, 13\right), 176(72), 117(100), 77$ (59).

Preparation of (E)-methyl 2-(2-(bromomethyl)phenyl)-3-methoxyacrylate (7). To a mixture of $14.4 \mathrm{~g}$ (70 mmol) of (E)-methyl 3-methoxy-2-o-tolylacrylate 4 and $13.7 \mathrm{~g}$ (77 mmol) of $\mathrm{N}$-bromosuccinimide (NBS) in $100 \mathrm{~mL}$ of anhydrous carbon tetrachloride was added catalytic amount of 2,2'-azo-bisisobutyronitrile (AIBN). The resulting solution was refluxed for $12 \mathrm{~h}$, then cooled and filtered to remove succinimide. The filtrate was evaporated under a reduced pressure, and the residue was subjected to column chromatography using a mixture of petroleum ether and

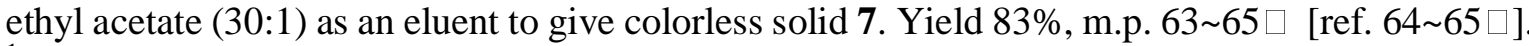
${ }^{1} \mathrm{H} \mathrm{NMR}\left(\mathrm{CDCl}_{3}\right): \delta 3.68\left(\mathrm{~s}, 3 \mathrm{H}, \mathrm{COOCH}_{3}\right), 3.81\left(\mathrm{~s}, 3 \mathrm{H}, \mathrm{OCH}_{3}\right), 4.40\left(\mathrm{~s}, 2 \mathrm{H}, \mathrm{CH}_{2}\right), 7.09-7.52(\mathrm{~m}$, 4H, ArH), 7.64 (s, 1H, C=CH). EI MS: m/z (\%) $284\left(\mathrm{M}^{+}, 11\right), 253$ (12), 205 (23), 173 (38), 145 (100).

Preparation of substituted chalcones (8). To a mixture of appropriate benzaldehyde (15 mmol) and appropriate acetophenone $(15 \mathrm{mmol})$ in $60 \mathrm{~mL}$ of anhydrous ethanol was added $0.6 \mathrm{~g}(15$ mmol) of sodium hydroxide. The resulting solution was stirred for $24 \mathrm{~h}$ at room temp,erature. Then, poured into water, and neutralized with dilute $\mathrm{HCl}$. The resulting precipitate was filtered off and recrystallized from ethanol to give light yellow crystals.<smiles>Cc1ccc(/C=C/C(=O)c2cccc(O)c2)cc1</smiles>

Data for 8a: Yield, 83\%. mp, 110-112 $\square .{ }^{1} \mathrm{H}$ NMR (400 MHz, $\mathrm{CDCl}_{3}$ ): $\delta: 2.39$ (s, 3H, $\mathrm{CH}_{3}$ ), 7.12-7.25 (m, 3H, ArH ), 7.35-7.45 (m, 2H, ArH), 7.49-7.67 (m, 4H, ArH, =CH-CO), 7.79 (d, J = $15.6 \mathrm{~Hz}, 1 \mathrm{H},=\mathrm{CH}-\mathrm{Ar})$. EI MS m/z (\%): $238\left(\mathrm{M}^{+}\right)$.<smiles>COc1ccc(/C=C/C(=O)c2cccc(O)c2)cc1</smiles>

Data for 8b: Yield, 79\%. mp, 93-95 $\square .{ }^{1} \mathrm{H}$ NMR (400 MHz, $\left.\mathrm{CDCl}_{3}\right)$ : $\delta: 3.91$ (s, 3H, Ar- $\mathrm{OCH}_{3}$ ), $6.92(\mathrm{~d}, J=6.6 \mathrm{~Hz}, 2 \mathrm{H}, \mathrm{ArH}), 7.10(\mathrm{~d}, J=8.4 \mathrm{~Hz}, 1 \mathrm{H}, \mathrm{ArH}), 7.32-7.38$ (m, 2H, ArH), 7.54-7.63 $(\mathrm{m}, 4 \mathrm{H}, \mathrm{ArH},=\mathrm{CH}-\mathrm{CO}), 7.79(\mathrm{~d}, J=15.6 \mathrm{~Hz}, 1 \mathrm{H},=\mathrm{CH}-\mathrm{Ar})$. EI MS m/z (\%): $254\left(\mathrm{M}^{+}\right)$.

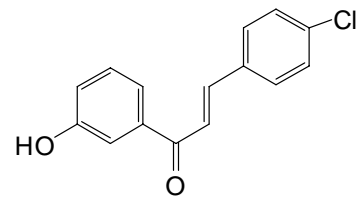

Data for 8c: Yield, 87\%. mp, 148-151 $\square .{ }^{1} \mathrm{H} \mathrm{NMR}\left(400 \mathrm{MHz}, \mathrm{CDCl}_{3}\right): \delta: 7.08(\mathrm{~d}, J=6.6 \mathrm{~Hz}, 2 \mathrm{H}$, ArH ), 7.16 (d, J=9.2 Hz, 1H, ArH), 7.37-7.44 (m, 2H, ArH), 7.54-7.63 (m, 4H, ArH, =CH-CO), $7.80(\mathrm{~d}, J=15.6 \mathrm{~Hz}, 1 \mathrm{H},=\mathrm{CH}-\mathrm{Ar})$. EI MS $m / z(\%): 258\left(\mathrm{M}^{+}\right)$. 


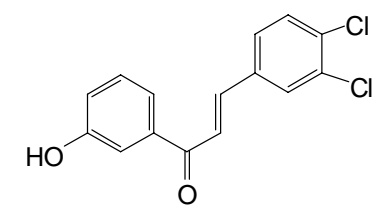

Data for 8d: Yield, 85\%. mp, 93-96 $\square .{ }^{1} \mathrm{H}$ NMR (400 MHz, $\left.\mathrm{CDCl}_{3}\right): \delta: 6.98(\mathrm{~d}, J=8.8 \mathrm{~Hz}, 2 \mathrm{H}$, $\mathrm{ArH}), 7.26-7.68(\mathrm{~m}, 5 \mathrm{H}, \mathrm{ArH},=\mathrm{CH}-\mathrm{CO}), 7.81-7.87(\mathrm{~m}, 2 \mathrm{H}, \mathrm{ArH},=\mathrm{CH}-\mathrm{Ar})$. EI MS: $m / z(\%): 292$ $\left(\mathrm{M}^{+}\right)$.

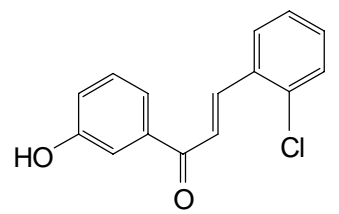

Data for 8e: Yield, 92\%. mp, 119-121 $\square .{ }^{1} \mathrm{H}$ NMR $\left(400 \mathrm{MHz}, \mathrm{CDCl}_{3}\right): \delta: 6.91(\mathrm{~d}, J=8.4 \mathrm{~Hz}, 2 \mathrm{H}$, $\mathrm{ArH}$ ), 7.10-7.16 (m, 2H, ArH), 7.38 (s, 1H, ArH), 7.64-7.84 (m, 3H, ArH, =CH-CO), 7.92-7.97 $(\mathrm{m}, 2 \mathrm{H}, \mathrm{ArH},=\mathrm{CH}-\mathrm{Ar})$. EI MS m/z (\%): $258\left(\mathrm{M}^{+}\right)$.<smiles>O=C(CCC1CCCC(Cl)C1)c1cccc(O)c1</smiles>

Data for 8f: Yield, 88\%. mp, 156-158 $\square .{ }^{1} \mathrm{H}$ NMR $\left(400 \mathrm{MHz}, \mathrm{CDCl}_{3}\right)$ : $8: 7.08-7.11(\mathrm{~m}, 1 \mathrm{H}, \mathrm{ArH})$, 7.30-7.35 (m, 1H, ArH), 7.39 (s, 1H, ArH), 7.47-7.56 (m, 6H, ArH, =CH-CO), $7.72(\mathrm{~d}, J=16.0$ $\mathrm{Hz}, 1 \mathrm{H},=\mathrm{CH}-\mathrm{Ar})$. EI MS $m / z(\%): 258\left(\mathrm{M}^{+}\right)$.<smiles>O=C(/C=C/c1ccc(F)cc1)c1cccc(O)c1</smiles>

Data for 8g: Yield, 89\%. mp, 124-126 $\square .{ }^{1} \mathrm{H}$ NMR (400 MHz, $\left.\mathrm{CDCl}_{3}\right)$ : $\delta: 7.10-7.17$ (m, 3H, $\mathrm{ArH})$, 7.39-7.47 (m, 2H, ArH), 7.55-7.64 (m, 4H, ArH, =CH-CO), $7.81(\mathrm{~d}, J=16.4 \mathrm{~Hz}, 1 \mathrm{H}$, $=$ CH-Ar). EI MS $m / z(\%): 242\left(\mathrm{M}^{+}\right)$.<smiles>O=C(/C=C/c1ccccc1)c1cccc(O)c1</smiles>

Data for 8h: Yield, 93\%. mp, 130-132 $\square .{ }^{1} \mathrm{H}$ NMR $\left(400 \mathrm{MHz}, \mathrm{CDCl}_{3}\right): \delta: 6.95(\mathrm{~d}, J=6.6 \mathrm{~Hz}, 2 \mathrm{H}$, $\mathrm{ArH}$ ), $7.08(\mathrm{~d}, J=8.4 \mathrm{~Hz}, 1 \mathrm{H}, \mathrm{ArH}), 7.32-7.39(\mathrm{~m}, 2 \mathrm{H}, \mathrm{ArH}), 7.54-7.62(\mathrm{~m}, 5 \mathrm{H}, \mathrm{ArH},=\mathrm{CH}-\mathrm{CO})$, $7.76(\mathrm{~d}, J=16.0 \mathrm{~Hz}, 1 \mathrm{H},=\mathrm{CH}-\mathrm{Ar})$. EI MS $m / z(\%): 224\left(\mathrm{M}^{+}\right)$

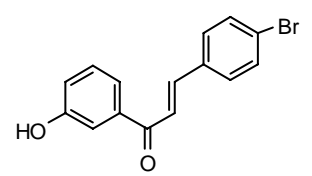

Data for 8i: Yield, 87\%. mp, 143-145 $\square .{ }^{1} \mathrm{H}$ NMR (400 MHz, $\left.\mathrm{CDCl}_{3}\right)$ : $8: 7.08-7.12(\mathrm{~m}, 1 \mathrm{H}, \mathrm{ArH})$, 7.31-7.40 (m, 2H, ArH), 7.45-7.56 (m, 6H, ArH, =CH-CO), 7.77 (d, $J=16.0 \mathrm{~Hz}, 1 \mathrm{H},=\mathrm{CH}-\mathrm{Ar})$. EI MS $m / z(\%): 303\left(\mathrm{M}^{+}\right)$. 
<smiles>Cc1cccc(/C=C/C(=O)c2cccc(O)c2)c1</smiles>

Data for 8j: Yield, 94\%. mp, 89-91 $\square .{ }^{1} \mathrm{H}$ NMR (400 MHz, $\mathrm{CDCl}_{3}$ ): $\delta: 2.40$ (s, 3H, $\mathrm{CH}_{3}$ ), 7.10-7.24 (m, 2H, ArH), 7.29-7.44 (m, 4H, ArH), 7.48-7.60 (m, 3H, ArH, =CH-CO), 7.76 (d, $J=$ $15.6 \mathrm{~Hz}, 1 \mathrm{H},=\mathrm{CH}-\mathrm{Ar}) ; \mathrm{MS} \mathrm{m} / z(\%): 238\left(\mathrm{M}^{+}\right)$.<smiles>COc1ccc(/C=C/C(=O)c2cccc(O)c2)cc1OC</smiles>

Data for 8k: Yield, 90\%. mp, 123-126 $\square .{ }^{1} \mathrm{H}$ NMR (400 MHz, $\left.\mathrm{CDCl}_{3}\right): \delta: 3.86\left(\mathrm{~s}, 3 \mathrm{H}, \mathrm{OCH}_{3}\right.$ ), 3.90 (s, 3H, $\left.\mathrm{OCH}_{3}\right), 6.90-7.12(\mathrm{~m}, 2 \mathrm{H}, \mathrm{ArH}), 7.44-7.61$ (m, 5H, ArH, =CH-CO), 7.71-7.79 (m, $2 \mathrm{H}, \mathrm{ArH},=\mathrm{CH}-\mathrm{Ar})$. EI MS: $\mathrm{m} / z(\%) 284\left(\mathrm{M}^{+}\right)$.<smiles>O=C(/C=C/c1cccc(Br)c1)c1cccc(O)c1</smiles>

Data for 81: Yield, 85\%. mp, 131-133 $\square .{ }^{1} \mathrm{H}$ NMR (400 MHz, $\mathrm{CDCl}_{3}$ ): $\delta: 7.09-7.13$ (m, 1H, ArH), 7.32-7.37 (m, 1H, ArH), $7.40(\mathrm{~s}, 1 \mathrm{H}, \mathrm{ArH}), 7.47-7.56(\mathrm{~m}, 6 \mathrm{H}, \mathrm{ArH},=\mathrm{CH}-\mathrm{CO}), 7.78$ (d, J = 15.6 $\mathrm{Hz}, 1 \mathrm{H},=\mathrm{CH}-\mathrm{Ar})$. EI MS $m / z(\%): 303\left(\mathrm{M}^{+}\right)$.<smiles>Cc1ccc(/C=C/C(=O)c2cccc(O)c2)cc1C</smiles>

Data for 8m: Yield, 88\%. mp, 129-130 $\square .{ }^{1} \mathrm{H}$ NMR (400 MHz, $\left.\mathrm{CDCl}_{3}\right): \delta: 2.23\left(\mathrm{~s}, 3 \mathrm{H}, \mathrm{CH}_{3}\right)$, 2.25(s, 3H, $\left.\mathrm{CH}_{3}\right), 6.93-7.12(\mathrm{~m}, 2 \mathrm{H}, \mathrm{ArH}), 7.46-7.63$ (m, 5H, ArH, =CH-CO), 7.71-7.81 (m, 2H, $\mathrm{ArH},=\mathrm{CH}-\mathrm{Ar})$. EI MS: $m / z(\%) 252\left(\mathrm{M}^{+}\right)$.

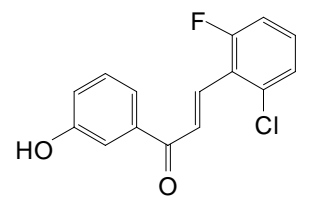

Data for 8n: Yield, 91\%. mp, 158-160 $\square .{ }^{1} \mathrm{H}$ NMR (400 MHz, $\mathrm{CDCl}_{3}$ ): $\delta:$ 7.10-7.21 (m, 2H, ArH), 7.28-7.41(m, 3H, ArH), 7.55-7.61 (m, 3H, ArH, $=\mathrm{CH}-\mathrm{CO}), 8.00(\mathrm{~d}, J=16.4 \mathrm{~Hz}, 1 \mathrm{H}$, $=\mathrm{CH}-\mathrm{Ar}) ; \mathrm{EI} \mathrm{MS}: \mathrm{m} / z(\%) 276\left(\mathrm{M}^{+}\right)$.<smiles>CN(C)c1ccc(/C=C/C(=O)c2cccc(O)c2)cc1</smiles>

Data for 8o: Yield, 87\%. mp, 170-172 $\square .{ }^{1} \mathrm{H}$ NMR (400 MHz, $\left.\mathrm{CDCl}_{3}\right): \delta: 3.04\left(\mathrm{~s}, 6 \mathrm{H}, 2 \times \mathrm{CH}_{3}\right)$, $6.70(\mathrm{~d}, J=8.8 \mathrm{~Hz}, 2 \mathrm{H}, \mathrm{ArH}), 7.10-7.22(\mathrm{~m}, 2 \mathrm{H}, \mathrm{ArH}), 7.29-7.58(\mathrm{~m}, 5 \mathrm{H}, \mathrm{ArH},=\mathrm{CH}-\mathrm{CO}), 7.77$ (d, $J=16.0 \mathrm{~Hz}, 1 \mathrm{H},=\mathrm{CH}-\mathrm{Ar})$; EI MS: $m / z(\%) 267\left(\mathrm{M}^{+}\right)$. 
<smiles>O=C(/C=C/c1ccc2c(c1)OCO2)c1cccc(O)c1</smiles>

Data for 8p: Yield, 77\%. mp, 179-181 $\square .{ }^{1} \mathrm{H}$ NMR (400 MHz, $\mathrm{CDCl}_{3}$ ): $\delta: 6.03$ (s, 2H, $\mathrm{OCH}_{2} \mathrm{O}$ ), $6.85(\mathrm{~d}, J=7.2 \mathrm{~Hz}, 1 \mathrm{H}, \mathrm{ArH}), 7.09-7.21(\mathrm{~m}, 2 \mathrm{H}, \mathrm{ArH}), 7.27-7.67(\mathrm{~m}, 5 \mathrm{H}, \mathrm{ArH},=\mathrm{CH}-\mathrm{CO}), 7.74$ $(\mathrm{d}, J=15.6 \mathrm{~Hz}, 1 \mathrm{H},=\mathrm{CH}-\mathrm{Ar})$; EI MS: $m / z(\%) 268\left(\mathrm{M}^{+}\right)$.<smiles>O=C(/C=C/c1ccco1)c1cccc(O)c1</smiles>

Data for 8q: Yield, 78\%. mp, 118-120 $\square .{ }^{1} \mathrm{H}$ NMR (400 MHz, $\mathrm{CDCl}_{3}$ ): $\delta: 6.52$ (s, 1H, ArH), 6.73 $(\mathrm{d}, J=1.8 \mathrm{~Hz}, 1 \mathrm{H}, \mathrm{ArH}), 7.11-7.20$ (m, 2H, ArH), 7.32-7.38(m, 3H, ArH), 7.52-7.64 (m, 2H, ArH, $=\mathrm{CH}-\mathrm{CO},=\mathrm{CH}-\mathrm{Ar}) ; \mathrm{EI} \mathrm{MS:} m / z(\%) 214\left(\mathrm{M}^{+}\right)$.<smiles>CCOc1ccc(/C=C/C(=O)c2cccc(O)c2)cc1</smiles>

Data for 8r: Yield, 93\%. mp, 116-118 $\square .{ }^{1} \mathrm{H}$ NMR (400 MHz, $\left.\mathrm{CDCl}_{3}\right): \delta: 1.43(\mathrm{t}, J=6.8 \mathrm{~Hz}, 3 \mathrm{H}$, $\left.\mathrm{CH}_{3}\right), 4.08\left(\mathrm{dd}, J_{1}=7.2 \mathrm{~Hz}, J_{2}=14.0 \mathrm{~Hz}, 2 \mathrm{H}, \mathrm{CH}_{2} \mathrm{CH}_{3}\right), 6.92-6.98(\mathrm{~m}, 2 \mathrm{H}, \mathrm{ArH}), 7.21-7.42(\mathrm{~m}, 4 \mathrm{H}$, $\mathrm{ArH}), 7.56-7.71(\mathrm{~m}, 3 \mathrm{H}, \mathrm{ArH},=\mathrm{CH}-\mathrm{CO}), 7.80(\mathrm{~d}, J=15.6 \mathrm{~Hz}, 1 \mathrm{H},=\mathrm{CH}-\mathrm{Ar})$; EI MS: $m / z(\%) 268$ $\left(\mathrm{M}^{+}\right)$.<smiles>O=C(/C=C/c1ccc(Cl)cc1Cl)c1cccc(O)c1</smiles>

Data for 8s: Yield, 87\%. mp, 97-99 $\square .{ }^{1} \mathrm{H}$ NMR (400 MHz, $\left.\mathrm{CDCl}_{3}\right): \delta: 7.12-7.21(\mathrm{~m}, 2 \mathrm{H}, \mathrm{ArH})$, 7.29-7.47 (m, 3H, ArH), 7.53-7.66 (m, 3H, ArH, =CH-CO), 8.06 (d, J=15.6Hz, 1H, =CH-Ar); EI MS: $m / z(\%) 292\left(\mathbf{M}^{+}\right)$.<smiles>O=C(/C=C/c1cccs1)c1cccc(O)c1</smiles>

Data for 8t: Yield, 81\%. mp, 122-124 $\square .{ }^{1} \mathrm{H}$ NMR (400 MHz, $\mathrm{CDCl}_{3}$ ): $\delta: 7.09-7.13$ (m, 2H, ArH), 7.29-7.44(m, 4H, ArH), 7.56-7.61 (m, 2H, ArH, =CH-CO), 7.94 (d, J=15.2Hz, 1H, =CH-Ar); EI MS: $m / z(\%) 230\left(\mathrm{M}^{+}\right)$.<smiles>O=C(/C=C/c1cccc(F)c1)c1cccc(O)c1</smiles>

Data for 1u: Yield, 89\%. mp, 121-123 $\square .{ }^{1} \mathrm{H}$ NMR (400 MHz, $\mathrm{CDCl}_{3}$ ): $\delta: 7.09-7.14$ (m, 2H, ArH), 7.32-7.37 (m, 4H, ArH), 7.44 (s, 1H, ArH), 7.51-7.57 (m, 2H, ArH, =CH-CO), 7.79 (d, J = 15.6 $\mathrm{Hz}, 1 \mathrm{H},=\mathrm{CH}-\mathrm{Ar})$. EI MS m/z (\%): $242\left(\mathrm{M}^{+}\right)$. 
<smiles>O=C(/C=C/c1ccccc1F)c1cccc(O)c1</smiles>

Data for 1v: Yield, 83\%. mp, 87-89 $\square .{ }^{1} \mathrm{H}$ NMR (400 MHz, $\left.\mathrm{CDCl}_{3}\right): \delta: 7.03(\mathrm{~d}, J=4.4 \mathrm{~Hz}, 2 \mathrm{H}$, ArH ), 7.12-7.18 (m, 2H, ArH), 7.38 (s, 1H, ArH), 7.66-7.74 (m, 3H, ArH, =CH-CO), 7.81 (d, $J=$ $15.6 \mathrm{~Hz}, 2 \mathrm{H}, \mathrm{ArH},=\mathrm{CH}-\mathrm{Ar})$. EI MS: $\mathrm{m} / z(\%) 242\left(\mathrm{M}^{+}\right)$.<smiles>O=C(/C=C/c1ccccc1Br)c1cccc(O)c1</smiles>

Data for 1w: Yield, 86\%. mp, 98-100 $\square .{ }^{1} \mathrm{H}$ NMR (400 MHz, $\left.\mathrm{CDCl}_{3}\right)$ : $\delta: 6.96(\mathrm{~d}, \mathrm{~J}=4.2 \mathrm{~Hz}, 2 \mathrm{H}$, ArH ), 7.12-7.19 (m, 2H, ArH), 7.39 (s, 1H, ArH), 7.62-7.81 (m, 3H, ArH, =CH-CO), 7.75-7.78 $(\mathrm{m}, 2 \mathrm{H}, \mathrm{ArH},=\mathrm{CH}-\mathrm{Ar})$. EI MS: $\mathrm{m} / z(\%) 303\left(\mathbf{M}^{+}\right)$.<smiles>O=C(/C=C/c1ccc(CI)cc1)c1cccc(O)c1</smiles>

Data for 8x: Yield, 88\%. mp, 86-88 $\square .{ }^{1} \mathrm{H}$ NMR (400 MHz, $\left.\mathrm{CDCl}_{3}\right): \delta: 1.21(\mathrm{t}, J=6.8 \mathrm{~Hz}, 3 \mathrm{H}$, $\left.\mathrm{CH}_{3}\right), 1.44\left(\mathrm{dd}, J_{1}=6.8 \mathrm{~Hz}, J_{2}=12.8 \mathrm{~Hz}, 2 \mathrm{H}, \mathrm{CH}_{2} \mathrm{CH}_{3}\right), 6.93(\mathrm{~d}, J=4.2 \mathrm{~Hz}, 2 \mathrm{H}, \mathrm{ArH}), 7.05-7.21$ $(\mathrm{m}, 2 \mathrm{H}, \mathrm{ArH}), 7.32-7.39(\mathrm{~m}, 3 \mathrm{H}, \mathrm{ArH}), 7.54-7.60(\mathrm{~m}, 2 \mathrm{H}, \mathrm{ArH},=\mathrm{CH}-\mathrm{CO}), 7.78(\mathrm{~d}, J=16.4 \mathrm{~Hz}$, $1 \mathrm{H},=\mathrm{CH}-\mathrm{Ar})$. EI MS: $m / z(\%) 252\left(\mathrm{M}^{+}\right)$.<smiles>CC(C)(C)c1ccc(/C=C/C(=O)c2cccc(O)c2)cc1</smiles>

Data for 8y: Yield, 87\%. mp, 139-141 $\square .{ }^{1} \mathrm{H}$ NMR (400 MHz, $\left.\mathrm{CDCl}_{3}\right): \delta: 1.34\left(\mathrm{~s}, 9 \mathrm{H}, 3 \times \mathrm{CH}_{3}\right)$, 7.08-7.21 (m, 2H, ArH), 7.32-7.46 (m, 4H, ArH), 7.55-7.61 (m, 3H, ArH, =CH-CO), 7.78 (d, $J=$ $16.0 \mathrm{~Hz}, 1 \mathrm{H},=\mathrm{CH}-\mathrm{Ar})$; EI MS: $m / z(\%) 280\left(\mathrm{M}^{+}\right)$.

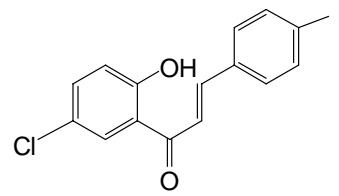

Data for 8aa: Yield, 78\%. mp, 121-124 $\square .{ }^{1} \mathrm{H}$ NMR (400 MHz, DMSO-d 6 ): $\delta: 2.34$ (s, 3H, $\mathrm{CH}_{3}$ ), 6.94-7.01 (m, 2H, ArH), 7.44-7.76 (m, 6H, ArH, =CH-CO), 7.97 (d, J=16.0 Hz, 1H, =CH-Ar), 12.75 (s, 1H, OH). EI MS: $m / z(\%): 272\left(\mathrm{M}^{+}\right)$.

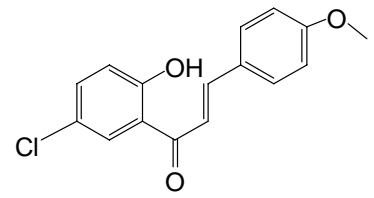

Data for 8bb: Yield, 74\%. mp, 88-90 $\square .{ }^{1} \mathrm{H}$ NMR (400 MHz, DMSO-d 6 ): $\delta: 3.85$ (s, 3H, $\mathrm{OCH}_{3}$ ), 6.91-7.02 (m, 2H, ArH), 7.43-7.63 (m, 6H, ArH, =CH-CO), 7.95 (d, J=16.0 Hz, 1H, =CH-Ar), $12.76(\mathrm{~s}, 1 \mathrm{H}, \mathrm{OH})$. EI MS: $m / z(\%): 288\left(\mathrm{M}^{+}\right)$. 


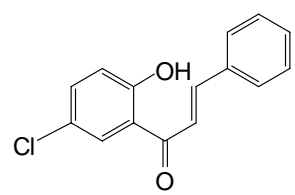

Data for 8cc: Yield, 66\%. mp, 99-101 $\square .{ }^{1} \mathrm{H}$ NMR (400 MHz, DMSO-d $\left.{ }_{6}\right): \delta: 6.91-7.02$ (m, 2H, ArH), 7.49-7.66 (m, 7H, ArH, =CH-CO), 7.90 (d, $J=16.0 \mathrm{~Hz}, 1 \mathrm{H},=\mathrm{CH}-\mathrm{Ar}), 12.76(\mathrm{~s}, 1 \mathrm{H}, \mathrm{OH})$. EI MS: $m / z(\%) 258\left(\mathrm{M}^{+}\right)$<smiles>OC(=CCc1cccc(Cl)c1)c1ccccc1</smiles>

Data for 8dd: Yield, 67\%. mp, 94-96 $\square .{ }^{1} \mathrm{H}$ NMR (400 MHz, DMSO-d 6 ): $\delta: 6.94-7.02$ (m, 2H, ArH), 7.52-7.67 (m, 7H, ArH, =CH-CO), 7.89 (d, J=15.6 Hz, 1H, =CH-Ar), 12.75 (s, 1H, OH). EI MS: $m / z(\%): 258\left(\mathrm{M}^{+}\right)$.<smiles>O=C(/C=C/c1cccc(Br)c1)c1ccccc1O</smiles>

Data for 8ee: Yield, 72\%. mp, 99-101 $\square .{ }^{1} \mathrm{H}$ NMR (400 MHz, DMSO-d 6 ): $\delta: 6.95-7.02$ (m, 2H, $\mathrm{ArH})$, 7.49-7.65 (m, 6H, ArH, =CH-CO), 7.83-7.93 (m, 2H, ArH, =CH-Ar), 12.68 (s, 1H, OH). EI MS: $m / z(\%): 303\left(\mathbf{M}^{+}\right)$.

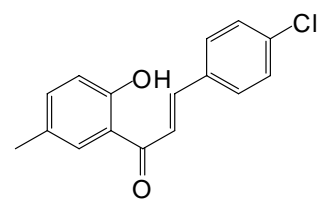

Data for 8ff: Yield, 78\%. mp, 116-118 $\square .{ }^{1} \mathrm{H}$ NMR (400 MHz, DMSO-d $\left.{ }_{6}\right): \delta: 2.32\left(\mathrm{~s}, 3 \mathrm{H}, \mathrm{CH}_{3}\right)$, 6.92-7.01 (m, 2H, ArH), 7.49-7.65 (m, 6H, ArH, =CH-CO), $7.88(\mathrm{~d}, J=15.6 \mathrm{~Hz}, 1 \mathrm{H},=\mathrm{CH}-\mathrm{Ar})$, 12.73 (s, 1H, OH). EI MS: $m / z(\%): 272\left(\mathrm{M}^{+}\right)$.<smiles>O=C(C=Cc1ccccc1Cl)c1cc(Cl)ccc1O</smiles>

Data for 8gg: Yield, 70\%. mp, 101-103 $\square .{ }^{1} \mathrm{H}$ NMR (400 MHz, DMSO-d $\left.{ }_{6}\right)$ : $\delta: 6.91-7.24$ (m, 2H, ArH), 7.57-7.72 (m, 5H, ArH, =CH-CO), 7.86-7.90 (m, 2H, ArH, =CH-Ar), $12.70(\mathrm{~s}, 1 \mathrm{H}, \mathrm{OH})$. EI MS $m / z(\%): 292\left(\mathrm{M}^{+}\right)$.

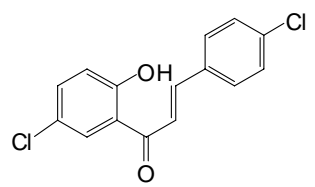

Data for 8hh: Yield, 71\%. mp, 186-189 $\square .{ }^{1} \mathrm{H}$ NMR (400 MHz, DMSO-d 6 ): $\delta: 6.89-7.04$ (m, 2H, ArH), 7.47-7.76 (m, 5H, ArH, =CH-CO), 7.84-7.90 (m, 2H, ArH, =CH-Ar), 12.72 (s, 1H, OH). EI MS $m / z(\%): 292\left(\mathrm{M}^{+}\right)$. 


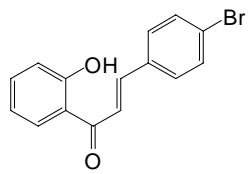

Data for 8i: Yield, 67\%. mp, 138-140 $\square .{ }^{1} \mathrm{H}$ NMR (400 MHz, DMSO-d 6 ): $\delta: 6.95-7.03$ (m, 2H, $\mathrm{ArH})$, 7.52-7.67 (m, 6H, ArH, =CH-CO), 7.83-7.92 (m, 2H, ArH, =CH-Ar), 12.75 (s, 1H, OH). EI MS: $m / z(\%): 303\left(\mathbf{M}^{+}\right)$.

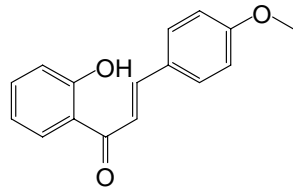

Data for 8jj: Yield, 78\%. mp, 83-85 $\square .{ }^{1} \mathrm{H}$ NMR (400 MHz, DMSO-d 6 ): $\delta: 3.98\left(\mathrm{~s}, 3 \mathrm{H}, \mathrm{OCH}_{3}\right)$, 6.92-7.06 (m, 2H, ArH), 7.52-7.62 (m, 6H, ArH, =CH-CO), 7.73-7.82 (m, 2H, ArH, =CH-Ar), $12.71(\mathrm{~s}, 1 \mathrm{H}, \mathrm{OH})$. EI MS: $m / z(\%): 254\left(\mathrm{M}^{+}\right)$.

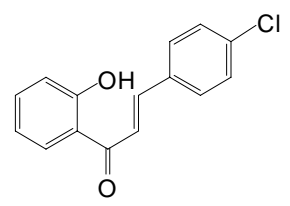

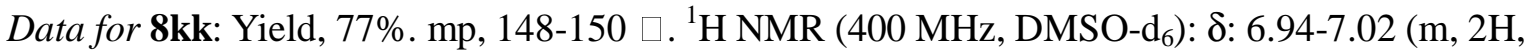
ArH), 7.52-7.97 (m, 7H, ArH, =CH-CO), 8.09 (d, $J=16.4 \mathrm{~Hz}, 1 \mathrm{H},=\mathrm{CH}-\mathrm{Ar}), 12.72(\mathrm{~s}, 1 \mathrm{H}, \mathrm{OH})$. EI MS: $m / z(\%): 258\left(\mathrm{M}^{+}\right)$.

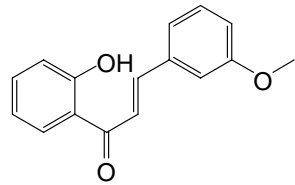

Data for 8ll: Yield, 74\%. mp, 88-90 $\square .{ }^{1} \mathrm{H}$ NMR (400 MHz, DMSO-d 6 ): $\delta: 3.98$ (s, $\left.3 \mathrm{H}, \mathrm{OCH}_{3}\right)$, 6.95-7.06 (m, 2H, ArH), 7.52-7.63 (m, 6H, ArH, =CH-CO), 7.72-7.82 (m, 2H, ArH, =CH-Ar), 12.68 (s, 1H, OH). EI MS: $m / z(\%): 254\left(\mathrm{M}^{+}\right)$.

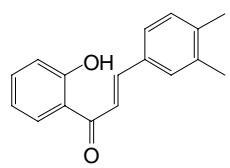

Data for 8mm: Yield, 65\%. mp, 110-112 $\square .{ }^{1} \mathrm{H}$ NMR (400 MHz, DMSO-d 6 ): $\delta: 2.23$ (s, 3H, $\left.\mathrm{CH}_{3}\right), 2.26\left(\mathrm{~s}, 3 \mathrm{H}, \mathrm{CH}_{3}\right), 6.93-7.12(\mathrm{~m}, 2 \mathrm{H}, \mathrm{ArH}), 7.46-7.63(\mathrm{~m}, 5 \mathrm{H}, \mathrm{ArH},=\mathrm{CH}-\mathrm{CO}), 7.71-7.80(\mathrm{~m}$, $2 \mathrm{H}, \mathrm{ArH},=\mathrm{CH}-\mathrm{Ar}), 12.70(\mathrm{~s}, 1 \mathrm{H}, \mathrm{OH})$. EI MS: $m / z(\%) 252\left(\mathrm{M}^{+}\right)$.<smiles>COc1ccc(/C=C/C(=O)c2ccccc2O)cc1OC</smiles>

Data for 8nn: Yield, 67\%. mp, 115-117 $\square .{ }^{1} \mathrm{H}$ NMR (400 MHz, DMSO-d ${ }_{6}$ ): $\delta: 3.89$ (s, 3H, $\left.\mathrm{OCH}_{3}\right), 3.91\left(\mathrm{~s}, 3 \mathrm{H}, \mathrm{OCH}_{3}\right), 6.90-7.11(\mathrm{~m}, 2 \mathrm{H}, \mathrm{ArH}), 7.46-7.61(\mathrm{~m}, 5 \mathrm{H}, \mathrm{ArH},=\mathrm{CH}-\mathrm{CO})$, 7.71-7.83 (m, 2H, ArH, =CH-Ar), $12.68(\mathrm{~s}, 1 \mathrm{H}, \mathrm{OH})$. EI MS: m/z (\%) $284\left(\mathrm{M}^{+}\right)$. 


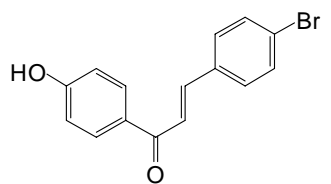

Data for 8aaa: Yield, 92\%. mp, 156-157 $\square .{ }^{1} \mathrm{H}$ NMR (400 MHz, $\left.\mathrm{CDCl}_{3}\right): \delta: 7.06(\mathrm{~d}, J=6.2 \mathrm{~Hz}$, 2H, ArH ), 7.12 (d, $J=4.2 \mathrm{~Hz}, 1 \mathrm{H}, \mathrm{ArH}), 7.31-7.43$ (m, 2H, ArH), 7.55-7.65 (m, 4H, ArH, $=\mathrm{CH}-\mathrm{CO}), 7.78(\mathrm{~d}, J=15.6 \mathrm{~Hz}, 1 \mathrm{H},=\mathrm{CH}-\mathrm{Ar})$. EI MS m/z $(\%): 304\left(\mathrm{M}^{+}+1\right)$.

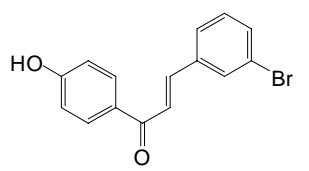

Data for 8bbb: Yield 87\%. mp, 127-130 $\square .{ }^{1} \mathrm{H}$ NMR (400 MHz, $\left.\mathrm{CDCl}_{3}\right)$ : $\delta: 7.05-7.11(\mathrm{~m}, 1 \mathrm{H}$, ArH), 7.31-7.37 (m, 1H, ArH), 7.41 (s, 1H, ArH), 7.47-7.56 (m, 6H, ArH, =CH-CO), 7.78 (d, $J=$ $16.0 \mathrm{~Hz}, 1 \mathrm{H},=\mathrm{CH}-\mathrm{Ar})$. EI MS m/z (\%): $304\left(\mathrm{M}^{+}+1\right)$.

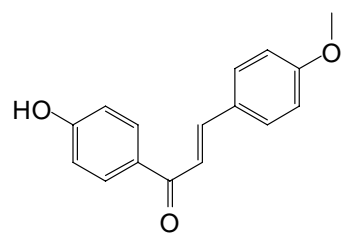

Data for 8ccc: Yield, 94\%. mp, 186-188 $\square .{ }^{1} \mathrm{H}$ NMR (400 MHz, $\left.\mathrm{CDCl}_{3}\right): \delta: 3.93$ (s, 3H, Ar-OCH $\left.{ }_{3}\right), 6.96(\mathrm{~d}, J=6.4 \mathrm{~Hz}, 2 \mathrm{H}, \mathrm{ArH}), 7.12(\mathrm{~d}, J=4.2 \mathrm{~Hz}, 1 \mathrm{H}, \mathrm{ArH}), 7.32-7.38$ (m, 2H, ArH), 7.54-7.63 (m, 4H, ArH, =CH-CO), 7.77 (d, $J=15.6 \mathrm{~Hz}, 1 \mathrm{H},=\mathrm{CH}-\mathrm{Ar})$. EI MS $\mathrm{m} / \mathrm{z}(\%): 255$ $\left(\mathrm{M}^{+}+1\right)$.

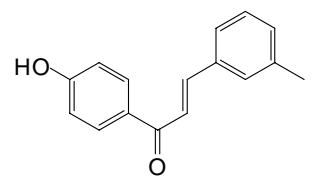

Data for 8ddd: Yield, 92\%. mp, 151-153 $\square .{ }^{1} \mathrm{H}$ NMR (400 MHz, $\left.\mathrm{CDCl}_{3}\right): \delta: 2.40\left(\mathrm{~s}, 3 \mathrm{H}, \mathrm{CH}_{3}\right)$, 6.93-6.95 (m, 2H, ArH), 7.22-7.30 (m, 2H, ArH), 7.42-7.56 (m, 3H, ArH, =CH-CO), 7.75 (d, J= $15.6 \mathrm{~Hz}, 1 \mathrm{H},=\mathrm{CH}-\mathrm{Ar}), 7.96-7.98(\mathrm{~m}, 2 \mathrm{H}, \mathrm{ArH})$. EI MS: $m / z(\%) 443\left([\mathrm{M}+1]^{+}, 5\right), 239\left(\mathrm{M}^{+}+1\right)$.

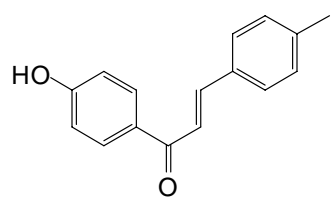

Data for 8eee: Yield, 89\%. mp, 189-192 $\square .{ }^{1} \mathrm{H}$ NMR (400 MHz, $\mathrm{CDCl}_{3}$ ): $\delta: 2.30\left(\mathrm{~s}, 3 \mathrm{H}, \mathrm{OCH}_{3}\right)$, $6.93(\mathrm{~d}, J=6.4 \mathrm{~Hz}, 2 \mathrm{H}, \mathrm{ArH}), 7.08$ (d, $J=4.0 \mathrm{~Hz}, 1 \mathrm{H}, \mathrm{ArH}), 7.32-7.37$ (m, 2H, ArH), 7.55-7.67 $(\mathrm{m}, 4 \mathrm{H}, \mathrm{ArH},=\mathrm{CH}-\mathrm{CO}), 7.79(\mathrm{~d}, J=15.6 \mathrm{~Hz}, 1 \mathrm{H},=\mathrm{CH}-\mathrm{Ar})$. EI MS m/z (\%): $239\left(\mathrm{M}^{+}+1\right)$.

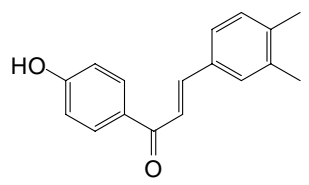

Data for 8fff: Yield, 92\%. mp, 152-155 $\square .{ }^{1} \mathrm{H}$ NMR (400 MHz, $\mathrm{CDCl}_{3}$ ): ${ }^{1} \mathrm{H}$ NMR (400 MHz, $\left.\mathrm{CDCl}_{3}\right): \delta: 2.21\left(\mathrm{~s}, 3 \mathrm{H}, \mathrm{CH}_{3}\right), 2.24\left(\mathrm{~s}, 3 \mathrm{H}, \mathrm{CH}_{3}\right), 6.93-7.12(\mathrm{~m}, 2 \mathrm{H}, \mathrm{ArH}), 7.46-7.63(\mathrm{~m}, 5 \mathrm{H}, \mathrm{ArH}$, $=\mathrm{CH}-\mathrm{CO}), 7.73-7.79(\mathrm{~m}, 2 \mathrm{H}, \mathrm{ArH},=\mathrm{CH}-\mathrm{Ar})$. EI MS: $m / z(\%) 253\left(\mathrm{M}^{+}+1\right)$. 


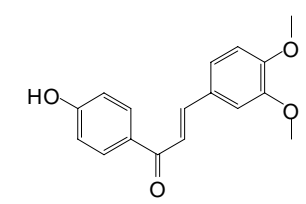

Data for 8ggg: Yield, 90\%. mp, 193-195 $\square .{ }^{1} \mathrm{H}$ NMR (400 MHz, $\mathrm{CDCl}_{3}$ ): $\delta: 3.93$ (s, 3H, $\mathrm{OCH}_{3}$ ), $3.95\left(\mathrm{~s}, 3 \mathrm{H}, \mathrm{OCH}_{3}\right), 6.86-6.95(\mathrm{~m}, 2 \mathrm{H}, \mathrm{ArH}), 7.15-7.26(\mathrm{~m}, 3 \mathrm{H}, \mathrm{ArH}), 7.34-7.66(\mathrm{~m}, 2 \mathrm{H}, \mathrm{ArH}$, $=\mathrm{CH}-\mathrm{CO}), 7.99-8.02(\mathrm{~m}, 2 \mathrm{H}, \mathrm{ArH},=\mathrm{CH}-\mathrm{Ar})$. EI MS: $\mathrm{m} / z(\%) 285\left(\mathrm{M}^{+}+1\right)$.

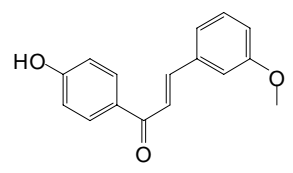

Data for 8hhh: Yield, 88\%. mp, 147-150 $\square .{ }^{1} \mathrm{H} \mathrm{NMR}\left(400 \mathrm{MHz}, \mathrm{CDCl}_{3}\right): \delta: 3.93\left(\mathrm{~s}, 3 \mathrm{H}, \mathrm{OCH}_{3}\right)$, 6.89-6.98 (m,2H, ArH), 7.12-7.29 (m, 4H, ArH), 7.34-7.77 (m, 2H, ArH, =CH-CO, =CH-Ar), $8.01(\mathrm{~d}, J=4.4 \mathrm{~Hz}, 2 \mathrm{H}, \mathrm{ArH})$. EI MS: $m / z(\%) 255\left(\mathrm{M}^{+}+1\right)$.

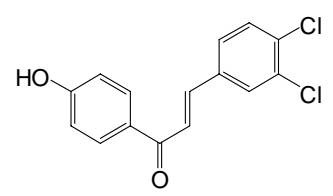

Data for 8iii: Yield, 91\%. mp, 142-144 $\square .{ }^{1} \mathrm{H}$ NMR (400 MHz, $\left.\mathrm{CDCl}_{3}\right): \delta: 6.97(\mathrm{~d}, J=4.4 \mathrm{~Hz}, 2 \mathrm{H}$, $\mathrm{ArH}), 7.26-7.71(\mathrm{~m}, 5 \mathrm{H}, \mathrm{ArH},=\mathrm{CH}-\mathrm{CO},=\mathrm{CH}-\mathrm{Ar}), 8.01(\mathrm{~d}, J=8.4 \mathrm{~Hz}, 2 \mathrm{H}, \mathrm{ArH})$. EI MS: $\mathrm{m} / z(\%)$ : $293\left(\mathrm{M}^{+}+1\right)$.

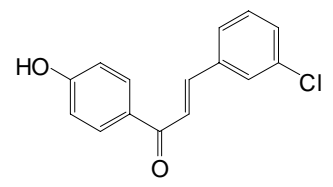

Data for 8jjj: Yield, 94\%. mp, 168-170 $\left.\square .{ }^{1} \mathrm{H} \mathrm{NMR} \mathrm{(400} \mathrm{MHz,} \mathrm{CDCl}_{3}\right): \delta: 6.99(\mathrm{~d}, J=4.4 \mathrm{~Hz}, 2 \mathrm{H}$, $\mathrm{ArH}), 7.18-7.49$ (m, 3H, ArH), 7.60-7.75(m, 2H, ArH, =CH-CO), 7.78 (d, $J=4.4 \mathrm{~Hz}, 2 \mathrm{H}, \mathrm{ArH})$, $8.12(\mathrm{~d}, J=15.6 \mathrm{~Hz}, 1 \mathrm{H},=\mathrm{CH}-\mathrm{Ar})$. EI MS: $m / z(\%) 259\left(\mathrm{M}^{+}+1\right)$.

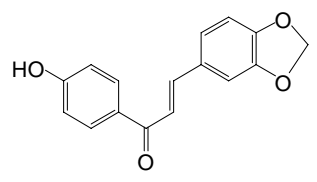

Data for 8kkk: Yield, 81\%. mp, 190-192 $\square .{ }^{1} \mathrm{H}$ NMR (400 MHz, $\left.\mathrm{CDCl}_{3}\right): \delta: 6.02\left(\mathrm{~s}, 2 \mathrm{H}, \mathrm{CH}_{2}\right)$, $6.85(\mathrm{~d}, J=8.4 \mathrm{~Hz}, 1 \mathrm{H}, \mathrm{ArH}), 6.98(\mathrm{~d}, J=8.8 \mathrm{~Hz}, 2 \mathrm{H}, \mathrm{ArH}), 7.18-7.44$ (m, 3H, ArH, =CH-CO), $7.69(\mathrm{~d}, J=8.0 \mathrm{~Hz}, 2 \mathrm{H}, \mathrm{ArH}), 7.99(\mathrm{~d}, J=15.6 \mathrm{~Hz}, 1 \mathrm{H},=\mathrm{CH}-\mathrm{Ar})$. EI MS: $m / z(\%) 269\left(\mathrm{M}^{+}+1\right)$.

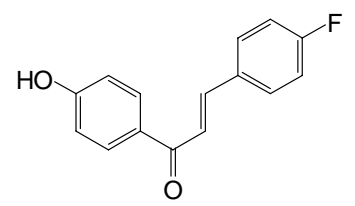

Data for 81ll: Yield, 89\%. mp, 161-163 $\left.\square .{ }^{1} \mathrm{H} \mathrm{NMR} \mathrm{(400} \mathrm{MHz,} \mathrm{CDCl}_{3}\right): \delta: 6.99(\mathrm{~d}, J=8.0 \mathrm{~Hz}, 2 \mathrm{H}$, ArH), 7.10-7.27 (m, 3H, ArH), 7.41-7.72 (m, 3H, ArH, =CH-CO), 7.98-8.01 (m, 2H, ArH, $=\mathrm{CH}-\mathrm{Ar})$. EI MS: $m / z(\%) 243\left(\mathrm{M}^{+}+1\right)$. 


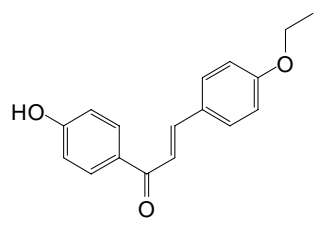

Data for 8mmm: Yield, 90\%. mp, 186-189 $\left.\square .{ }^{1} \mathrm{H} \mathrm{NMR} \mathrm{(400} \mathrm{MHz,} \mathrm{CDCl}_{3}\right): \delta: 1.43(\mathrm{t}, J=7.6 \mathrm{~Hz}$, $\left.3 \mathrm{H}, \mathrm{CH}_{3}\right), 4.08\left(\mathrm{dd}, J_{1}=7.2 \mathrm{~Hz}, J_{2}=13.2 \mathrm{~Hz}, 2 \mathrm{H}, \mathrm{CH}_{2} \mathrm{CH}_{3}\right), 6.92-6.98(\mathrm{~m}, 2 \mathrm{H}, \mathrm{ArH}), 7.23-7.41(\mathrm{~m}$, 2H, ArH), 7.51-7.78 (m, 4H, ArH, =CH-CO), 7.97-7.99 (m, 2H, ArH, =CH-Ar); EI MS: $m / z(\%)$ $269\left(\mathrm{M}^{+}+1\right)$.

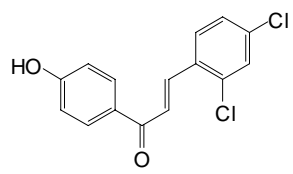

Data for 8nnn: Yield, 92\%. mp, 186-189 $\square .{ }^{1} \mathrm{H}$ NMR (400 MHz, $\left.\mathrm{CDCl}_{3}\right): \delta: 6.99(\mathrm{~d}, J=8.8 \mathrm{~Hz}$, 2H, ArH), 7.26-7.67 (m, 5H, ArH, =CH-CO), 7.95-8.10 (m, 2H, ArH, =CH-Ar). EI MS: $m / z(\%)$ $293\left(\mathrm{M}^{+}+1\right)$.

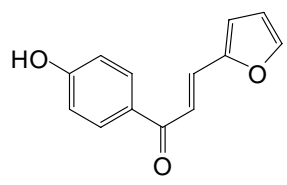

Data for 8ooo: Yield, 90\%. mp, 148-151 $\square .{ }^{1} \mathrm{H}$ NMR (400 MHz, $\mathrm{CDCl}_{3}$ ): $\delta: 6.50$ (s, $\left.1 \mathrm{H}, \mathrm{ArH}\right)$, 6.68 (s, 1H, ArH ), 6.99 (d, $J=8.4 \mathrm{~Hz}, 2 \mathrm{H}, \mathrm{ArH}), 7.37-7.59(\mathrm{~m}, 4 \mathrm{H}, \mathrm{ArH},=\mathrm{CH}-\mathrm{CO}), 8.01(\mathrm{~d}, J=$ $16.4 \mathrm{~Hz}, 1 \mathrm{H},=\mathrm{CH}-\mathrm{Ar})$. EI MS: $\mathrm{m} / z(\%) 215\left(\mathrm{M}^{+}+1\right)$.

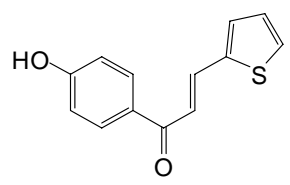

Data for 8ppp: Yield, 87\%. mp, 160-162 $\square .{ }^{1} \mathrm{H}$ NMR (400 MHz, $\left.\mathrm{CDCl}_{3}\right): \delta: 6.93(\mathrm{t}, 2 \mathrm{H}, J=$ $4.4 \mathrm{~Hz}, \mathrm{ArH}), 7.08(\mathrm{t}, 1 \mathrm{H}, J=1.8 \mathrm{~Hz}, \mathrm{ArH}), 7.29-7.44(\mathrm{~m}, 3 \mathrm{H}, \mathrm{ArH}), 7.89-7.96(\mathrm{~m}, 3 \mathrm{H}, \mathrm{ArH}$, $=\mathrm{CH}-\mathrm{CO},=\mathrm{CH}-\mathrm{Ar})$. EI MS: $m / z(\%) 231\left(\mathrm{M}^{+}+1\right)$.

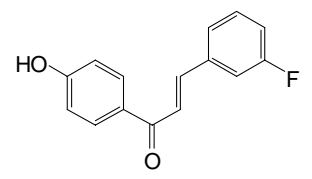

Data for 8qqq: Yield, 90\%. mp, 171-173 $\square .{ }^{1} \mathrm{H}$ NMR (400 MHz, $\mathrm{CDCl}_{3}$ ): $\delta: 6.99$ (d, J=8.0Hz, 2H, ArH), 7.16-7.32 (m, 3H, ArH), 7.46-7.77 (m, 3H, ArH, =CH-CO, =CH-Ar), 8.00 (d, $J=$ 4.4Hz, 2H, ArH). EI MS: $m / z(\%) 243\left(\mathrm{M}^{+}+1\right)$.

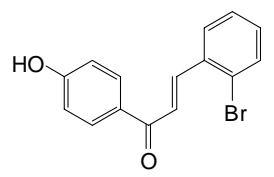

Data for 8rrr: Yield, 88\%. mp, 114-117 $\square .{ }^{1} \mathrm{H}$ NMR (400 MHz, $\left.\mathrm{CDCl}_{3}\right)$ : $\delta: 6.91(\mathrm{~d}, \mathrm{~J}=8.4 \mathrm{~Hz}$, $2 \mathrm{H}, \mathrm{ArH}), 7.02-7.11(\mathrm{~m}, 2 \mathrm{H}, \mathrm{ArH}), 7.36(\mathrm{~m}, 1 \mathrm{H}, \mathrm{ArH}), 7.51-7.76(\mathrm{~m}, 3 \mathrm{H}, \mathrm{ArH},=\mathrm{CH}-\mathrm{CO})$, 7.80-7.82 (m, 2H, ArH, =CH-Ar). EI MS: $m / z(\%) 304\left(\mathrm{M}^{+}+1\right)$. 


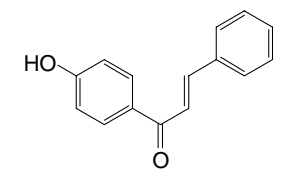

Data for 8sss: ${ }^{1} \mathrm{H}$ NMR (400 MHz, $\left.\mathrm{CDCl}_{3}\right)$ : $\delta: 6.94(\mathrm{~d}, J=8.8 \mathrm{~Hz}, 2 \mathrm{H}, \mathrm{ArH}), 7.40-7.57$ (m, 4H, $\mathrm{ArH}), 7.63-7.65$ (m, 2H, ArH, =CH-CO), $7.78(\mathrm{~d}, J=15.6 \mathrm{~Hz}, 1 \mathrm{H},=\mathrm{CH}-\mathrm{Ar}) .7 .97$ (d, $J=8.8 \mathrm{~Hz}$, 2H, ArH). EI MS: $m / z(\%) 224\left(\mathrm{M}^{+}\right)$.

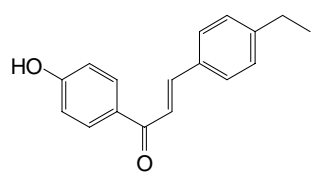

Data for 8ttt: Yield, 92\%. mp, 121-123 $\square .{ }^{1} \mathrm{H}$ NMR (400 MHz, $\left.\mathrm{CDCl}_{3}\right): \delta: 1.23(\mathrm{t}, J=7.8 \mathrm{~Hz}, 3 \mathrm{H}$, $\left.\mathrm{CH}_{3}\right), 1.44\left(\mathrm{dd}, J_{1}=6.4 \mathrm{~Hz}, J_{2}=12.8 \mathrm{~Hz}, 2 \mathrm{H}, \mathrm{CH}_{2} \mathrm{CH}_{3}\right), 6.93(\mathrm{~d}, J=8.4 \mathrm{~Hz}, 2 \mathrm{H}, \mathrm{ArH}), 7.02-7.21$ $(\mathrm{m}, 2 \mathrm{H}, \mathrm{ArH}), 7.31-7.39(\mathrm{~m}, 3 \mathrm{H}, \mathrm{ArH}), 7.54-7.63(\mathrm{~m}, 2 \mathrm{H}, \mathrm{ArH},=\mathrm{CH}-\mathrm{CO}), 7.78(\mathrm{~d}, J=16.8 \mathrm{~Hz}$, $1 \mathrm{H},=\mathrm{CH}-\mathrm{Ar})$. EI MS: $m / z(\%) 253\left(\mathrm{M}^{+}+1\right)$.

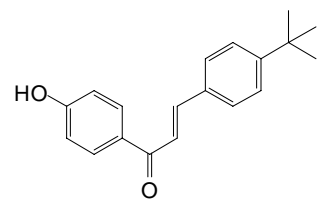

Data for 8uuu: Yield, 89\%. mp, 134-136 $\square .{ }^{1} \mathrm{H}$ NMR (400 MHz, $\left.\mathrm{CDCl}_{3}\right): \delta: 1.34\left(\mathrm{~s}, 9 \mathrm{H}, 3 \times \mathrm{CH}_{3}\right)$, 7.06-7.201 (m, 2H, ArH), 7.32-7.47 (m, 4H, ArH), 7.55-7.60(m, 3H, ArH, =CH-CO), 7.79 (d, $J=$ $16.0 \mathrm{~Hz}, 1 \mathrm{H},=\mathrm{CH}-\mathrm{Ar})$; EI MS: $m / z(\%) 281\left(\mathrm{M}^{+}+1\right)$.

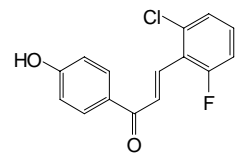

Data for 8vvv: Yield, 78\%. mp, 105-107 $\left.\square .{ }^{1} \mathrm{H} \mathrm{NMR} \mathrm{(400} \mathrm{MHz,} \mathrm{CDCl}_{3}\right): \delta: 6.99(\mathrm{~d}, J=8.0 \mathrm{~Hz}$, $2 \mathrm{H}, \mathrm{ArH}), 7.07-7.35$ (m, 2H, ArH), 7.50-7.61 (m, 3H, ArH, =CH-CO), 8.00-8.02 (m, 2H, ArH, $=\mathrm{CH}-\mathrm{Ar})$. EI MS: $m / z(\%) 277\left(\mathrm{M}^{+}+1\right)$.

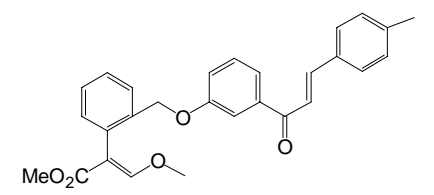

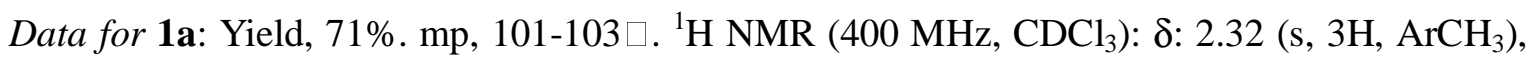
$3.70\left(\mathrm{~s}, 3 \mathrm{H}, \mathrm{COOCH}_{3}\right), 3.81\left(\mathrm{~s}, 3 \mathrm{H}, \mathrm{OCH}_{3}\right), 5.04\left(\mathrm{~s}, 2 \mathrm{H}, \mathrm{CH}_{2}\right), 7.14-7.23(\mathrm{~m}, 2 \mathrm{H}, \mathrm{ArH}), 7.33-7.44$ $(\mathrm{m}, 6 \mathrm{H}, \mathrm{ArH}), 7.51-7.59\left(\mathrm{~m}, 6 \mathrm{H}, \mathrm{ArH},=\mathrm{CH}-\mathrm{CO},=\mathrm{CH}_{-}-\mathrm{OCH}_{3}\right), 7.78(\mathrm{~d}, J=15.6 \mathrm{~Hz}, 1 \mathrm{H},=\mathrm{CH}-\mathrm{Ar})$. MS m/z (\%): $443\left(\mathrm{M}^{+}+1,24\right), 442\left(\mathrm{M}^{+}, 23\right), 236$ (96), 222 (100), 209 (47), 203 (93), 194 (72), 177 (94), 141 (89), 114 (98), 101 (95). Anal. Calcd for $\mathrm{C}_{28} \mathrm{H}_{26} \mathrm{O}_{5}$ : C, 76.00; H, 5.92. Found: C, $76.23 ; \mathrm{H}, 5.75$. 


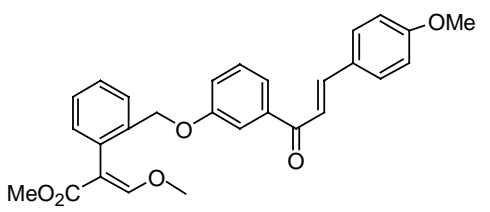

Data for 1b: Yield, 74\%. mp, 112-114 $\square .{ }^{1} \mathrm{H}$ NMR (400 MHz, $\left.\mathrm{CDCl}_{3}\right)$ : $\delta: 3.70\left(\mathrm{~s}, 3 \mathrm{H}, \mathrm{COOCH}_{3}\right)$, $3.81\left(\mathrm{~s}, 3 \mathrm{H}, \mathrm{OCH}_{3}\right), 3.91\left(\mathrm{~s}, 3 \mathrm{H}, \mathrm{Ar}-\mathrm{OCH}_{3}\right), 5.04\left(\mathrm{~s}, 2 \mathrm{H}, \mathrm{CH}_{2}\right), 6.92(\mathrm{~d}, J=9.2 \mathrm{~Hz}, 2 \mathrm{H}, \mathrm{ArH})$, 7.12 (t, $J=4.4 \mathrm{~Hz}, 1 \mathrm{H}, \mathrm{ArH}), 7.20-7.23$ (m, 1H, ArH), 7.32-7.38 (m, 4H, ArH), 7.54-7.59 (m, 6H, $\left.\mathrm{ArH},=\mathrm{CH}-\mathrm{CO},=\mathrm{C} \underline{\mathrm{H}}-\mathrm{OCH}_{3}\right), 7.77(\mathrm{~d}, J=15.2 \mathrm{~Hz}, 1 \mathrm{H},=\mathrm{CH}-\mathrm{Ar}) . \mathrm{MS} m / z(\%): 458\left(\mathrm{M}^{+}, 77\right), 426$ (M+ 66), 366 (21), 253 (99), 204 (100), 182 (38), 172 (36), 143 (94), 114(67), 102 (72). Anal. Calcd for $\mathrm{C}_{28} \mathrm{H}_{26} \mathrm{O}_{6}$ : C, 73.35; H, 5.72. Found: C, 73.14; H, 5.96.

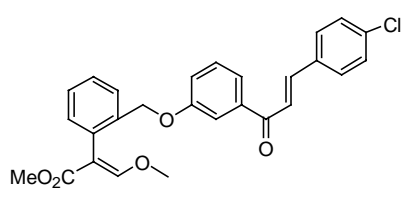

Data for 1c: Yield, 72\%. mp, 111-113 $\square .{ }^{1} \mathrm{H}$ NMR (400 MHz, $\mathrm{CDCl}_{3}$ ): $\delta: 3.70$ (s, 3H, $\mathrm{COOCH}_{3}$ ), $3.82\left(\mathrm{~s}, 3 \mathrm{H}, \mathrm{OCH}_{3}\right), 5.04\left(\mathrm{~s}, 2 \mathrm{H}, \mathrm{CH}_{2}\right), 7.13-7.21(\mathrm{~m}, 2 \mathrm{H}, \mathrm{ArH}), 7.34-7.42(\mathrm{~m}, 6 \mathrm{H}, \mathrm{ArH})$, 7.46-7.60 (m, 6H, ArH, $\left.=\mathrm{CH}-\mathrm{CO},=\mathrm{C} \underline{\mathrm{H}}-\mathrm{OCH}_{3}\right), 7.75(\mathrm{~d}, J=15.6 \mathrm{~Hz}, 1 \mathrm{H},=\mathrm{CH}-\mathrm{Ar}) . \mathrm{MS} m / z(\%)$ : $462\left(\mathrm{M}^{+}, 3\right), 205$ (45), 164 (41), 146 (27), 145 (100), 131 (33), 114 (35), 102 (27). Anal Calcd for $\mathrm{C}_{27} \mathrm{H}_{23} \mathrm{ClO}_{5}$ : C, 70.05; H, 5.01. Found: C, 69.77; H, 5.06.

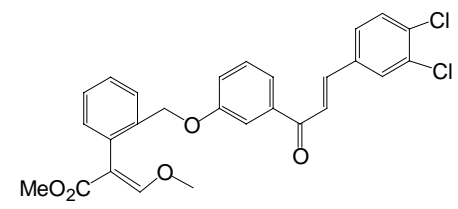

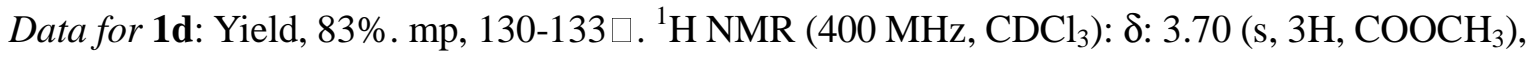
$3.82\left(\mathrm{~s}, 3 \mathrm{H}, \mathrm{OCH}_{3}\right), 5.04\left(\mathrm{~s}, 2 \mathrm{H}, \mathrm{CH}_{2}\right), 7.12-7.21(\mathrm{~m}, 2 \mathrm{H}, \mathrm{ArH}), 7.34-7.44(\mathrm{~m}, 5 \mathrm{H}, \mathrm{ArH})$, 7.47-7.67 (m, 6H, ArH, $\left.=\mathrm{CH}-\mathrm{CO},=\mathrm{CH}_{-}-\mathrm{OCH}_{3}\right), 7.87(\mathrm{~d}, J=16.0 \mathrm{~Hz}, 1 \mathrm{H},=\mathrm{CH}-\mathrm{Ar}) . \mathrm{MS} \mathrm{m} / \mathrm{z}(\%)$ : $496\left(\mathrm{M}^{+}, 3\right), 362$ (50), 330 (31), 204 (36), 171 (98), 162 (31), 144 (100), 132 (30), 119 (31), 101 (83). Anal. Calcd for $\mathrm{C}_{27} \mathrm{H}_{22} \mathrm{Cl}_{2} \mathrm{O}_{5}$ : C, 65.20; H, 4.46. Found: $\mathrm{C}, 65.39 ; \mathrm{H}, 4.28$.

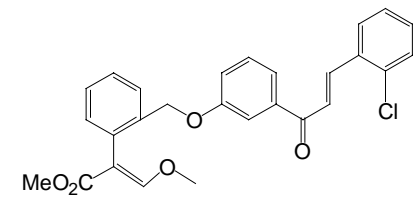

Data for 1e: Yield, 77\%. mp, 123-125 $\square .{ }^{1} \mathrm{H}$ NMR (400 MHz, $\left.\mathrm{CDCl}_{3}\right)$ : $\delta: 3.70\left(\mathrm{~s}, 3 \mathrm{H}, \mathrm{COOCH}_{3}\right)$, $3.82\left(\mathrm{~s}, 3 \mathrm{H}, \mathrm{OCH}_{3}\right), 5.04\left(\mathrm{~s}, 2 \mathrm{H}, \mathrm{CH}_{2}\right), 7.12-7.21(\mathrm{~m}, 2 \mathrm{H}, \mathrm{ArH}), 7.36-7.45(\mathrm{~m}, 7 \mathrm{H}, \mathrm{ArH})$, 7.55-7.61 (m, 4H, ArH, =CH-CO, =C $\underline{\mathrm{H}}-\mathrm{OCH} 3), 7.74(\mathrm{~d}, J=6.4 \mathrm{~Hz}, 1 \mathrm{H}, \mathrm{ArH}), 8.17(\mathrm{~d}, J=16.0$ $\mathrm{Hz}, 1 \mathrm{H},=\mathrm{CH}-\mathrm{Ar}) . \mathrm{MS} m / z(\%): 462\left(\mathrm{M}^{+}, 2\right), 222$ (12), 194 (30), 164 (35), 146 (31), 144 (100), 137 (21), 114 (46), 104 (18), 102 (55). Anal. Calcd for $\mathrm{C}_{27} \mathrm{H}_{23} \mathrm{ClO}_{5}$ : C, 70.05; H, 5.01. Found: C, 70.26; H, 5.29.

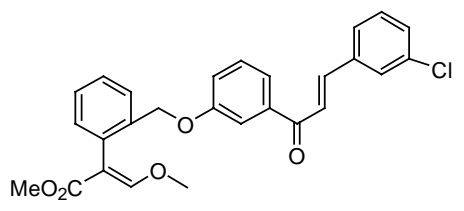

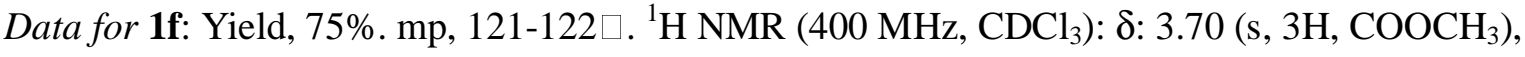


$3.82\left(\mathrm{~s}, 3 \mathrm{H}, \mathrm{OCH}_{3}\right), 5.04\left(\mathrm{~s}, 2 \mathrm{H}, \mathrm{CH}_{2}\right), 7.08-7.21(\mathrm{~m}, 2 \mathrm{H}, \mathrm{ArH}), 7.36-7.45(\mathrm{~m}, 7 \mathrm{H}, \operatorname{ArH})$, 7.55-7.61 (m, 4H, ArH, $\left.=\mathrm{CH}-\mathrm{CO},=\mathrm{CH}_{-}-\mathrm{OCH}_{3}\right), 7.91-7.99(\mathrm{~m}, 2 \mathrm{H}, \mathrm{ArH},=\mathrm{CH}-\mathrm{Ar}) . \mathrm{MS} \mathrm{m} / z(\%)$ : $462\left(\mathrm{M}^{+}, 3\right), 328$ (27), 204 (19), 188 (44), 161 (24), 144 (37), 138 (100), 118 (30), 101 (39). Anal. Calcd for $\mathrm{C}_{27} \mathrm{H}_{23} \mathrm{ClO}_{5}$ : C, 70.05; H, 5.01. Found: C, 70.14; H, 5.05.

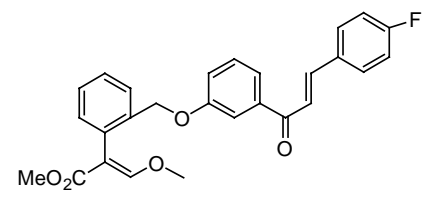

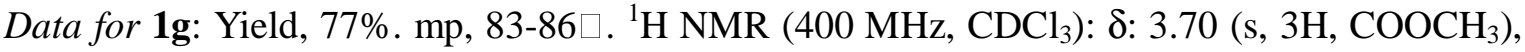
$3.82\left(\mathrm{~s}, 3 \mathrm{H}, \mathrm{OCH}_{3}\right), 5.04\left(\mathrm{~s}, 2 \mathrm{H}, \mathrm{CH}_{2}\right), 7.08-7.20(\mathrm{~m}, 4 \mathrm{H}, \mathrm{ArH}), 7.33-7.40(\mathrm{~m}, 4 \mathrm{H}, \mathrm{ArH})$, 7.53-7.63 (m, 6H, ArH, =CH-CO, =CH-OCH 3 ), $7.76(\mathrm{~d}, J=15.6 \mathrm{~Hz}, 1 \mathrm{H},=\mathrm{CH}-\mathrm{Ar}) . \mathrm{MS} \mathrm{m} / \mathrm{z}(\%)$ : 447 (M+13), 414 (29), 240 (98), 214 (30), 204 (98), 182 (86), 173 (93), 164 (44), 142 (90), 120 (100), 114(98). Anal. Calcd for $\mathrm{C}_{27} \mathrm{H}_{23} \mathrm{FO}_{5}$ : C, 72.63; H, 5.19. Found: C,72.89; H, 5.21.

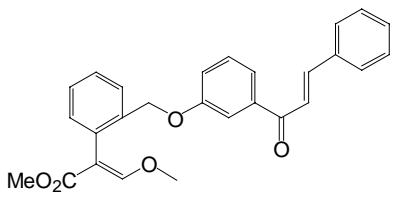

Data for 1h: Yield, 81\%. mp, 104-107 $\square .{ }^{1} \mathrm{H}$ NMR (400 MHz, $\left.\mathrm{CDCl}_{3}\right): \delta: 3.70$ (s, 3H, $\left.\mathrm{COOCH}_{3}\right), 3.81\left(\mathrm{~s}, 3 \mathrm{H}, \mathrm{OCH}_{3}\right), 5.04\left(\mathrm{~s}, 2 \mathrm{H}, \mathrm{CH}_{2}\right), 7.14-7.23(\mathrm{~m}, 2 \mathrm{H}, \mathrm{ArH}), 7.33-7.44(\mathrm{~m}, 6 \mathrm{H}$, $\mathrm{ArH})$, 7.46-7.65 (m, 6H, ArH, $\left.=\mathrm{CH}-\mathrm{CO},=\mathrm{CH}-\mathrm{OCH}_{3}\right), 7.77-7.82(\mathrm{~m}, 2 \mathrm{H}, \mathrm{ArH},=\mathrm{CH}-\mathrm{Ar}) . \quad \mathrm{MS}$ m/z (\%): 429 ( $\left.\mathrm{M}^{+}+1,18\right), 396$ (41), 222 (96), 204 (100), 194 (37), 177 (24), 144 (33), 129(84), 114 (49), 101(97). Anal Calcdfor $\mathrm{C}_{27} \mathrm{H}_{24} \mathrm{O}_{5}$ : C, 75.68; H, 5.65. Found: C, 75.89; H, 5.36.

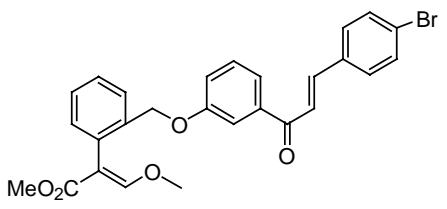

Data for 1i: Yield, 79\%. mp, 114-116 $\square .{ }^{1} \mathrm{H}$ NMR (400 MHz, $\left.\mathrm{CDCl}_{3}\right)$ : $\delta: 3.70$ (s, 3H, $\mathrm{COOCH}_{3}$ ), $3.82\left(\mathrm{~s}, 3 \mathrm{H}, \mathrm{OCH}_{3}\right), 5.04\left(\mathrm{~s}, 2 \mathrm{H}, \mathrm{CH}_{2}\right), 7.14-7.21(\mathrm{~m}, 2 \mathrm{H}, \mathrm{ArH}), 7.34-7.43(\mathrm{~m}, 6 \mathrm{H}, \mathrm{ArH})$, 7.47-7.60 (m, 6H, ArH, =CH-CO, =C $\left.\underline{\mathrm{H}}-\mathrm{OCH}_{3}\right), 7.74(\mathrm{~d}, J=15.6 \mathrm{~Hz}, 1 \mathrm{H},=\mathrm{CH}-\mathrm{Ar}) . \mathrm{MS} \mathrm{m} / z(\%)$ : $508\left(\mathrm{M}^{+}+1,4\right), 507\left(\mathrm{M}^{+}, 3\right), 204$ (16), 165 (13), 146 (31), 144 (100), 131(27), 114 (29), 102(36). Anal. Calcd for $\mathrm{C}_{27} \mathrm{H}_{23} \mathrm{BrO}_{5}$ : C, 63.92; H, 4.57. Found: C, 64.15; H, 4.41.<smiles>COc1cccc(Oc2cccc(C(=O)Cc3cccc(C)c3)c2)c1</smiles>

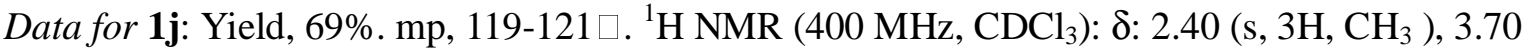
$\left(\mathrm{s}, 3 \mathrm{H}, \mathrm{COOCH}_{3}\right), 3.82\left(\mathrm{~s}, 3 \mathrm{H}, \mathrm{OCH}_{3}\right), 5.04\left(\mathrm{~s}, 2 \mathrm{H}, \mathrm{CH}_{2}\right), 7.11-7.24(\mathrm{~m}, 4 \mathrm{H}, \mathrm{ArH})$, 7.29-7.44 (m, 6H, ArH), 7.48-7.60 (m, 4H, ArH, =CH-CO, =C $\left.\underline{\mathrm{H}}-\mathrm{OCH}_{3}\right), 7.79$ (d, J = $\left.15.6 \mathrm{~Hz}, 1 \mathrm{H},=\mathrm{CH}-\mathrm{Ar}\right)$. MS m/z (\%): 442 (M+3), 205 (11), 189 (17), 165 (24), 145 (73), 144 (100), 131(44), 114 (89), 105 (45), 91 (100). Anal. Calcd for $\mathrm{C}_{28} \mathrm{H}_{26} \mathrm{O}_{5}$ : C, 76.00; H, 5.92. Found: C, 76.163; H, 5.97.

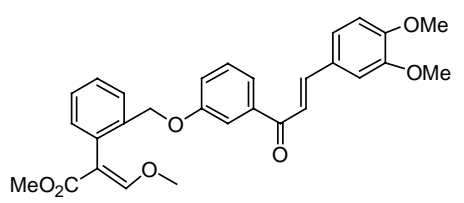

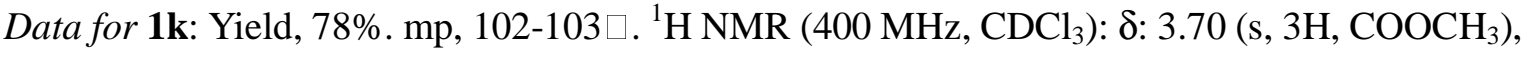


$3.81\left(\mathrm{~s}, 3 \mathrm{H}, \mathrm{OCH}_{3}\right), 3.93\left(\mathrm{~s}, 3 \mathrm{H}, \mathrm{Ar}-\mathrm{OCH}_{3}\right), 3.95$ (s, 3H, Ar-OCH $), 5.04$ (s, 2H, CH$), 6.90(\mathrm{~d}, J$ $=4.2 \mathrm{~Hz}, 1 \mathrm{H}, \mathrm{ArH}), 7.15-7.20(\mathrm{~m}, 4 \mathrm{H}, \mathrm{ArH}), 7.31-7.37(\mathrm{~m}, 4 \mathrm{H}, \mathrm{ArH}), 7.54-7.59(\mathrm{~m}, 4 \mathrm{H}, \mathrm{ArH}$, $\left.=\mathrm{CH}-\mathrm{CO},=\mathrm{C} \underline{\mathrm{H}}-\mathrm{OCH}_{3}\right), 7.76(\mathrm{~d}, J=15.6 \mathrm{~Hz}, 1 \mathrm{H},=\mathrm{CH}-\mathrm{Ar}) . \mathrm{EI} \mathrm{MS:} m / z(\%) 489\left([\mathrm{M}+1]^{+}, 6\right), 488$ $\left(\mathrm{M}^{+}, 25\right), 145$ (100), 144 (95), 131 (53), 114 (67), 102 (90). Anal. Calcd for $\mathrm{C}_{29} \mathrm{H}_{28} \mathrm{O}_{7}$ : C, 71.30 ; H, 5.78 . Found: C, 71.21; H,6.05.

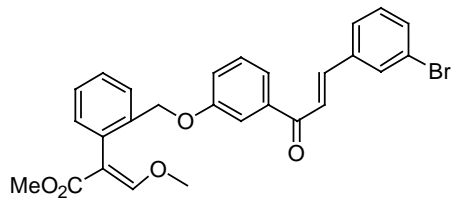

Data for 1l: Yield, 79\%. mp, 136-139 $\square .{ }^{1} \mathrm{H}$ NMR (400 MHz, $\mathrm{CDCl}_{3}$ ): $\delta: 3.70$ (s, $3 \mathrm{H}, \mathrm{COOCH}_{3}$ ), $3.82\left(\mathrm{~s}, 3 \mathrm{H}, \mathrm{OCH}_{3}\right), 5.04\left(\mathrm{~s}, 2 \mathrm{H}, \mathrm{CH}_{2}\right), 7.13-7.23(\mathrm{~m}, 2 \mathrm{H}, \mathrm{ArH}), 7.31-743(\mathrm{~m}, 7 \mathrm{H}, \mathrm{ArH})$,

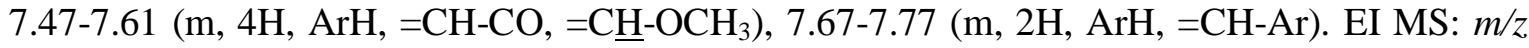
(\%) $508\left([\mathrm{M}+1]^{+}, 3\right), 507\left(\mathrm{M}^{+}, 2\right), 355$ (23), 220 (21), 187 (25), 148 (100), 146 (45), 104 (24), 91 (31). Anal. Calcd for $\mathrm{C}_{27} \mathrm{H}_{23} \mathrm{BrO}_{5}$ : C, 63.92; H, 4.57. Found: C, 64.13; H, 4.70.

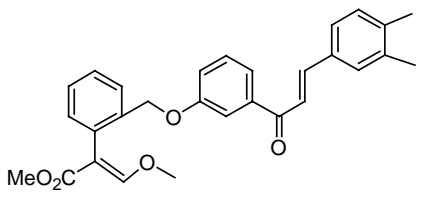

Data for 1m: Yield, 82\%. mp, 110-112.${ }^{1} \mathrm{H}$ NMR (400 MHz, $\mathrm{CDCl}_{3}$ ): $\delta: 2.30\left(\mathrm{~s}, 6 \mathrm{H}, 2 \times \mathrm{CH}_{3}\right.$ ), $3.70\left(\mathrm{~s}, 3 \mathrm{H}, \mathrm{COOCH}_{3}\right), 3.82\left(\mathrm{~s}, 3 \mathrm{H}, \mathrm{OCH}_{3}\right), 5.04\left(\mathrm{~s}, 2 \mathrm{H}, \mathrm{CH}_{2}\right), 7.11-7.21(\mathrm{~m}, 3 \mathrm{H}, \mathrm{ArH}), 7.34-7.45$ (m, 6H, ArH), 7.55-7.60 (m, 4H, ArH, =CH-CO, =C $\left.\underline{\mathrm{H}}-\mathrm{OCH}_{3}\right), 7.78(\mathrm{~d}, \mathrm{~J}=16.0 \mathrm{~Hz}, 1 \mathrm{H},=\mathrm{CH}-\mathrm{Ar})$. MS m/z (\%): $458\left(\mathrm{M}^{+}+2,8\right), 456\left(\mathrm{M}^{+}, 7\right), 250$ (12), 237 (66), 205 (39), 165 (18), 145 (100), 114 (68), 102 (47). Anal. Calcd for $\mathrm{C}_{29} \mathrm{H}_{28} \mathrm{O}_{5}$ : C, 76.30; H, 6.18. Found: C, 76.16; H,6.36.

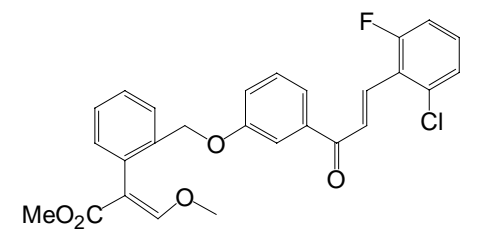

Data for 1n: Yield, 76\%. mp, 140-142 $\square .{ }^{1} \mathrm{H}$ NMR (400 MHz, $\left.\mathrm{CDCl}_{3}\right)$ : $\delta: 3.70\left(\mathrm{~s}, 3 \mathrm{H}, \mathrm{COOCH}_{3}\right.$ ), $3.84\left(\mathrm{~s}, 3 \mathrm{H}, \mathrm{OCH}_{3}\right), 5.04\left(\mathrm{~s}, 2 \mathrm{H}, \mathrm{CH}_{2}\right), 7.12-7.20(\mathrm{~m}, 3 \mathrm{H}, \mathrm{ArH}), 7.28-7.41(\mathrm{~m}, 5 \mathrm{H}, \mathrm{ArH})$, 7.55-7.61 (m, 4H, ArH, =CH-CO, =CH-OCH 3$), 7.79(\mathrm{~d}, J=8.0 \mathrm{~Hz}, 1 \mathrm{H}, \mathrm{ArH}), 8.01(\mathrm{~d}, J=16.4$ $\mathrm{Hz}, 1 \mathrm{H},=\mathrm{CH}-\mathrm{Ar})$. EI MS: m/z (\%) $480\left(\mathrm{M}^{+}, 5\right), 241$ (13), 205 (32), 204 (64), 146 (18), 145 (100), 144 (49), 114 (11), 102 (10). Anal. Calcd for $\mathrm{C}_{27} \mathrm{H}_{22} \mathrm{ClFO}_{5}$ : C, 67.43; H, 4.61. Found: C, 67.69; H, 4.37 .

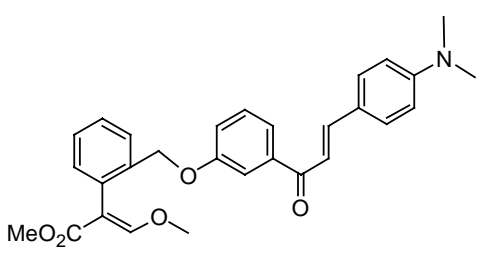

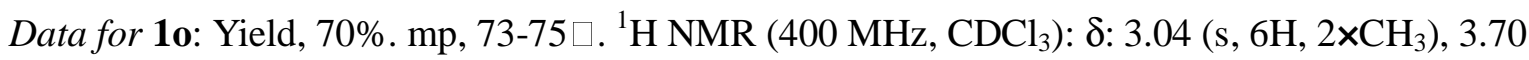
$\left(\mathrm{s}, 3 \mathrm{H}, \mathrm{COOCH}_{3}\right), 3.81\left(\mathrm{~s}, 3 \mathrm{H}, \mathrm{OCH}_{3}\right), 5.04\left(\mathrm{~s}, 2 \mathrm{H}, \mathrm{CH}_{2}\right), 6.70(\mathrm{~d}, J=4.2 \mathrm{~Hz}, 2 \mathrm{H}, \mathrm{ArH})$, 7.10-7.20 (m, 2H, ArH), 7.29-7.38(m, 4H, ArH), 7.52-7.60 (m, 6H, ArH, =CH-CO, =C $7.79(\mathrm{~d}, J=15.2 \mathrm{~Hz}, 1 \mathrm{H},=\mathrm{CH}-\mathrm{Ar})$. EI MS: $m / z(\%) 472\left([\mathrm{M}+1]^{+}, 7\right), 471\left(\mathrm{M}^{+}, 43\right), 265(15), 205$ (16), 204 (11), 145 (100), 114 (27), 102 (35), 101 (28). Anal. Calcd for $\mathrm{C}_{27} \mathrm{H}_{23} \mathrm{BrO}_{5}$ : C, 63.92; $\mathrm{H}$, 
4.57. Found: C, 63.99; H, 4.30.

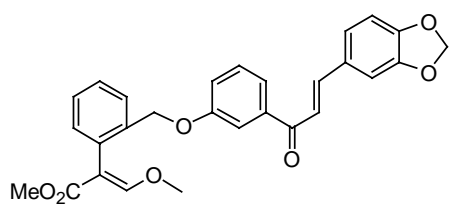

Data for 1p: Yield, 74\%. mp, 116-118 $\square .{ }^{1} \mathrm{H}$ NMR (400 MHz, $\mathrm{CDCl}_{3}$ ): $\delta: 3.70\left(\mathrm{~s}, 3 \mathrm{H}, \mathrm{COOCH}_{3}\right.$ ), $3.82\left(\mathrm{~s}, 3 \mathrm{H}, \mathrm{OCH}_{3}\right), 5.04\left(\mathrm{~s}, 2 \mathrm{H}, \mathrm{CH}_{2}\right), 6.03\left(\mathrm{~s}, 2 \mathrm{H}, \mathrm{OCH}_{2} \mathrm{O}\right), 6.85(\mathrm{~d}, J=7.2 \mathrm{~Hz}, 1 \mathrm{H}, \operatorname{ArH})$, 7.09-7.21 (m, 4H, ArH), 7.27-7.37(m, 4H, ArH), 7.52-7.60 (m, 4H, ArH, =CH-CO, =C $\underline{H}-\mathrm{OCH}_{3}$ ), $7.73(\mathrm{~d}, J=15.6 \mathrm{~Hz}, 1 \mathrm{H},=\mathrm{CH}-\mathrm{Ar})$. Anal. Calcd for $\mathrm{C}_{28} \mathrm{H}_{24} \mathrm{O}_{7}$ : C, 71.18; H, 5.12. Found: C, 71.29; H, 5.30 .<smiles>CO/C=C(/C)c1ccccc1COc1cccc(C(=O)/C=C/c2ccco2)c1</smiles>

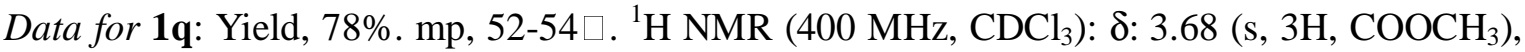
$3.80\left(\mathrm{~s}, 3 \mathrm{H}, \mathrm{OCH}_{3}\right), 5.04\left(\mathrm{~s}, 2 \mathrm{H}, \mathrm{CH}_{2}\right), 6.52(\mathrm{~s}, 1 \mathrm{H}, \mathrm{ArH}), 6.72(\mathrm{~d}, J=3.6 \mathrm{~Hz}, 1 \mathrm{H}, \mathrm{ArH})$, 7.12-7.20 (m, 2H, ArH), 7.32-7.43(m, 4H, ArH), 7.52-7.62 (m, 6H, ArH, =CH-CO, =C $\underline{\mathrm{H}}-\mathrm{OCH}_{3}$,

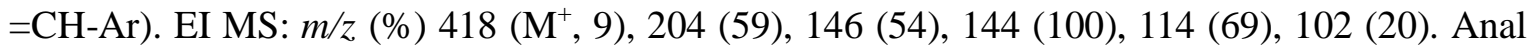
Calcd for $\mathrm{C}_{25} \mathrm{H}_{22} \mathrm{O}_{6}$ : C, 71.76; H, 5.30. Found: C, 71.61; H, 5.03.

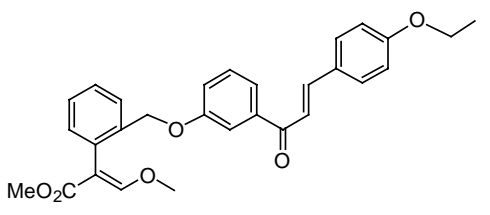

Data for 1r: Yield, 84\%. mp, 114-116.${ }^{1} \mathrm{H}$ NMR (400 MHz, $\left.\mathrm{CDCl}_{3}\right): \delta: 1.43(\mathrm{t}, J=6.8 \mathrm{~Hz}, 3 \mathrm{H}$, $\left.\mathrm{CH}_{3}\right), 3.70\left(\mathrm{~s}, 3 \mathrm{H}, \mathrm{COOCH}_{3}\right), 3.83\left(\mathrm{~s}, 3 \mathrm{H}, \mathrm{OCH}_{3}\right), 4.08\left(\mathrm{dd}, J_{1}=7.6 \mathrm{~Hz}, J_{2}=12.2 \mathrm{~Hz}, 2 \mathrm{H}\right.$, $\left.\mathrm{CH}_{2} \mathrm{CH}_{3}\right), 5.04$ (s, 2H, CH$\left.{ }_{2}\right), 6.93(\mathrm{~d}, J=8.4 \mathrm{~Hz}, 2 \mathrm{H}, \mathrm{ArH}), 7.10-7.21(\mathrm{~m}, 2 \mathrm{H}, \mathrm{ArH}), 7.33-7.39$ (m, $4 \mathrm{H}, \mathrm{ArH}), 7.54-7.60\left(\mathrm{~m}, 6 \mathrm{H}, \mathrm{ArH},=\mathrm{CH}-\mathrm{CO},=\mathrm{C} \underline{\mathrm{H}}-\mathrm{OCH}_{3}\right), 7.78(\mathrm{~d}, J=15.6 \mathrm{~Hz}, 1 \mathrm{H},=\mathrm{CH}-\mathrm{Ar}) . \mathrm{EI}$ MS: $m / z(\%) 473\left([\mathrm{M}+1]^{+}, 6\right), 472\left(\mathrm{M}^{+}, 6\right), 204$ (99), 146 (25), 145 (100), 144 (74), 131 (21). Anal. Calcd for $\mathrm{C}_{29} \mathrm{H}_{28} \mathrm{O}_{6}$ : C, 73.71; H, 5.97. Found: C, 73.90; H, 6.18.

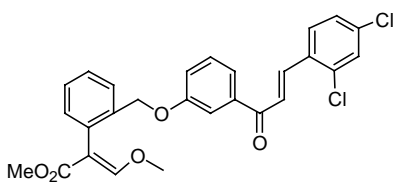

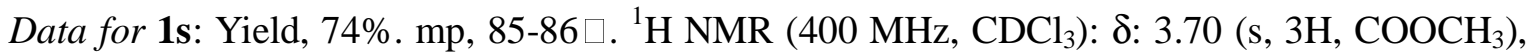
$3.83\left(\mathrm{~s}, 3 \mathrm{H}, \mathrm{OCH}_{3}\right), 5.04\left(\mathrm{~s}, 2 \mathrm{H}, \mathrm{CH}_{2}\right), 7.15-7.21(\mathrm{~m}, 2 \mathrm{H}, \mathrm{ArH}), 7.29-7.47(\mathrm{~m}, 6 \mathrm{H}, \mathrm{ArH})$, 7.53-7.60 (m, 4H, ArH, =CH-CO, =C $\underline{\mathrm{H}}-\mathrm{OCH} 3), 7.68(\mathrm{~d}, J=8.4 \mathrm{~Hz}, 1 \mathrm{H}, \mathrm{ArH}), 8.11(\mathrm{~d}, J=15.6 \mathrm{~Hz}$, 1H, =CH-Ar). EI MS: m/z (\%) $497\left(\mathrm{M}^{+}, 5\right), 317$ (41), 145 (100), 144 (92), 114 (27), 102 (38). Anal Calcd for $\mathrm{C}_{27} \mathrm{H}_{22} \mathrm{Cl}_{2} \mathrm{O}_{5}$ : C, 65.20; H, 4.46. Found: C, 65.49; H, 4.71.

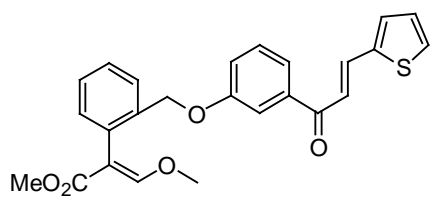

Data for 1t: Yield, 78\%. mp, 59-62 $\square .{ }^{1} \mathrm{H}$ NMR (400 MHz, $\left.\mathrm{CDCl}_{3}\right): \delta: 3.71\left(\mathrm{~s}, 3 \mathrm{H}, \mathrm{COOCH}_{3}\right)$, $3.83\left(\mathrm{~s}, 3 \mathrm{H}, \mathrm{OCH}_{3}\right), 5.03\left(\mathrm{~s}, 2 \mathrm{H}, \mathrm{CH}_{2}\right), 7.09-7.21(\mathrm{~m}, 3 \mathrm{H}, \mathrm{ArH}), 7.29-7.43(\mathrm{~m}, 6 \mathrm{H}, \mathrm{ArH})$, 
7.55-7.60 (m, 4H, ArH, $\left.=\mathrm{CH}-\mathrm{CO},=\mathrm{C} \underline{\mathrm{H}}-\mathrm{OCH}_{3}\right), 7.94(\mathrm{~d}, J=15.2 \mathrm{~Hz}, 1 \mathrm{H},=\mathrm{CH}-\mathrm{Ar}) . \mathrm{EI} \mathrm{MS}: \mathrm{m} / z$ (\%) $435\left([\mathrm{M}+1]^{+}, 6\right), 434\left(\mathrm{M}^{+}, 7\right), 205$ (88), 204 (44), 145 (100), 144 (72), 114 (12). Anal. Calcd for $\mathrm{C}_{25} \mathrm{H}_{22} \mathrm{O}_{5} \mathrm{~S}: \mathrm{C}, 69.11 ; \mathrm{H}, 5.10 ; \mathrm{S}, 7.38$. Found: C, 69.17; H, 5.03. S, 7.62.

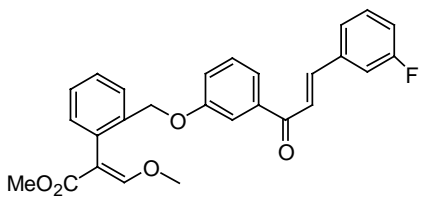

Data for 1u: Yield, 79\%. mp, 58-61 $\square .{ }^{1} \mathrm{H}$ NMR (400 MHz, $\left.\mathrm{CDCl}_{3}\right): \delta: 3.70\left(\mathrm{~s}, 3 \mathrm{H}, \mathrm{COOCH}_{3}\right)$, $3.82\left(\mathrm{~s}, 3 \mathrm{H}, \mathrm{OCH}_{3}\right), 5.05$ (s, 2H, $\mathrm{CH}_{2}$ ), 7.13-7.22 (m, 3H, ArH), 7.34-7.43 (m, 6H, ArH), 7.47 (s, $1 \mathrm{H}, \mathrm{ArH}), 7.53-7.60\left(\mathrm{~m}, 4 \mathrm{H}, \mathrm{ArH},=\mathrm{CH}-\mathrm{CO},=\mathrm{C} \underline{\mathrm{H}}-\mathrm{OCH}_{3}\right), 7.75(\mathrm{~d}, J=15.6 \mathrm{~Hz}, 1 \mathrm{H},=\mathrm{CH}-\mathrm{Ar}) . \mathrm{EI}$ MS: $m / z(\%) 447\left([\mathrm{M}+1]^{+}, 6\right), 446\left(\mathrm{M}^{+}, 10\right), 205$ (41), 204 (58), 145 (89), 144 (100), 102 (13). Anal. Calcd for $\mathrm{C}_{27} \mathrm{H}_{23} \mathrm{FO}_{5}$ : C, 72.63; H, 5.19. Found: C,72.92; H, 5.45..

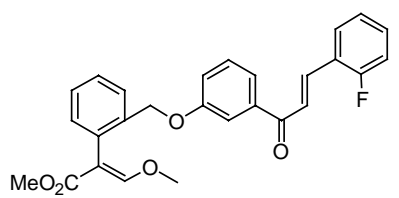

Data for 1v: Yield, 71\%. mp, 86-89 $\square .{ }^{1} \mathrm{H}$ NMR (400 MHz, $\left.\mathrm{CDCl}_{3}\right): \delta: 3.70\left(\mathrm{~s}, 3 \mathrm{H}, \mathrm{COOCH}_{3}\right)$, $3.83\left(\mathrm{~s}, 3 \mathrm{H}, \mathrm{OCH}_{3}\right), 5.04\left(\mathrm{~s}, 2 \mathrm{H}, \mathrm{CH}_{2}\right), 7.13-7.22(\mathrm{~m}, 2 \mathrm{H}, \mathrm{ArH}), 7.34-7.41(\mathrm{~m}, 4 \mathrm{H}, \mathrm{ArH})$, 7.53-7.63 (m, 8H, ArH, =CH-CO, =Cㅌ- $\left.-\mathrm{OCH}_{3}\right), 7.88(\mathrm{~d}, J=16.8 \mathrm{~Hz}, 1 \mathrm{H},=\mathrm{CH}-\mathrm{Ar})$. EI MS: $\mathrm{m} / \mathrm{z}$ (\%) $446\left(\mathrm{M}^{+}, 11\right), 241$ (38), 204 (76), 145 (99), 130 (65), 101 (100). Anal. Calcd for $\mathrm{C}_{27} \mathrm{H}_{23} \mathrm{FO}_{5}$ : C, 72.63; H, 5.19. Found: C,72.41; H, 5.12.

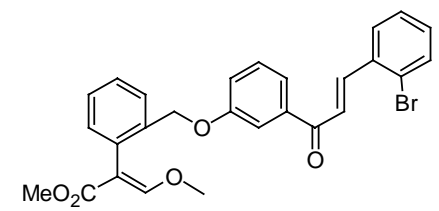

Data for 1w: Yield, 73\%. mp, 127-130 $\square .{ }^{1} \mathrm{H}$ NMR (400 MHz, $\left.\mathrm{CDCl}_{3}\right): \delta: 3.70\left(\mathrm{~s}, 3 \mathrm{H}, \mathrm{COOCH}_{3}\right)$, $3.82\left(\mathrm{~s}, 3 \mathrm{H}, \mathrm{OCH}_{3}\right), 5.05\left(\mathrm{~s}, 2 \mathrm{H}, \mathrm{CH}_{2}\right), 7.15-7.20(\mathrm{~m}, 2 \mathrm{H}, \mathrm{ArH}), 7.35-7.40(\mathrm{~m}, 6 \mathrm{H}, \operatorname{ArH})$, 7.55-7.65 (m, 5H, ArH, =CH-CO, =Cㅌ- $\left.-\mathrm{OCH}_{3}\right), 7.71(\mathrm{~d}, J=8.0 \mathrm{~Hz}, 1 \mathrm{H}, \mathrm{ArH}), 8.14(\mathrm{~d}, J=15.6$ $\mathrm{Hz}, 1 \mathrm{H},=\mathrm{CH}-\mathrm{Ar})$. EI MS: m/z (\%) $508\left([\mathrm{M}+1]^{+}, 3\right), 507\left(\mathrm{M}^{+}, 2\right), 205$ (68), 146 (36), 145 (100), 144 (76), 114 (21). Anal. Calcd for $\mathrm{C}_{27} \mathrm{H}_{23} \mathrm{BrO}_{5}$ : C, 63.92; H, 4.57. Found: $\mathrm{C}, 64.20 ; \mathrm{H}, 4.75$.

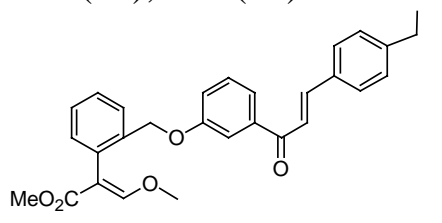

Data for 1x: Yield, 78\%. mp, 101-103 $\square .{ }^{1} \mathrm{H}$ NMR (400 MHz, $\left.\mathrm{CDCl}_{3}\right): \delta: 1.26(\mathrm{t}, J=7.6 \mathrm{~Hz}, 3 \mathrm{H}$, $\left.\mathrm{CH}_{3}\right), 1.44\left(\mathrm{dd}, J_{1}=7.6 \mathrm{~Hz}, J_{2}=12.8 \mathrm{~Hz}, 2 \mathrm{H}, \mathrm{CH}_{2} \mathrm{CH}_{3}\right), 3.70\left(\mathrm{~s}, 3 \mathrm{H}, \mathrm{COOCH}_{3}\right), 3.82(\mathrm{~s}, 3 \mathrm{H}$, $\left.\mathrm{OCH}_{3}\right), 5.04\left(\mathrm{~s}, 2 \mathrm{H}, \mathrm{CH}_{2}\right), 7.13-7.24(\mathrm{~m}, 4 \mathrm{H}, \mathrm{ArH}), 7.34-7.45$ (m, 6H, ArH), 7.56-7.60 (m, 4H, $\left.\mathrm{ArH},=\mathrm{CH}-\mathrm{CO},=\mathrm{CH}-\mathrm{OCH}_{3}\right), 7.78(\mathrm{~d}, J=15.6 \mathrm{~Hz}, 1 \mathrm{H},=\mathrm{CH}-\mathrm{Ar})$. EI MS: $m / z(\%) 457\left([\mathrm{M}+1]^{+}\right.$, 16), $456\left(\mathrm{M}^{+}, 27\right), 222$ (50), 205 (100), 204 (89), 145 (84), 144 (76), 102 (38). Anal. Calcd for $\mathrm{C}_{29} \mathrm{H}_{28} \mathrm{O}_{5}$ : C, 76.30; H, 6.18. Found: C,76.19; H, 6.43.

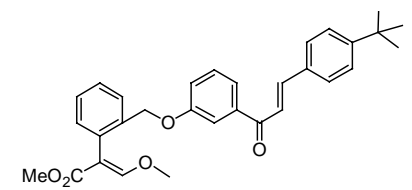


Data for 1y: Yield, 74\%. mp, 65-68 $\square .{ }^{1} \mathrm{H}$ NMR (400 MHz, $\left.\mathrm{CDCl}_{3}\right): \delta: 1.34\left(\mathrm{~s}, 9 \mathrm{H}, 3 \times \mathrm{CH}_{3}\right), 3.70$ $\left(\mathrm{s}, 3 \mathrm{H}, \mathrm{COOCH}_{3}\right), 3.82\left(\mathrm{~s}, 3 \mathrm{H}, \mathrm{OCH}_{3}\right), 5.04\left(\mathrm{~s}, 2 \mathrm{H}, \mathrm{CH}_{2}\right), 7.11-7.21(\mathrm{~m}, 2 \mathrm{H}, \mathrm{ArH}), 7.34-7.46(\mathrm{~m}$, $6 \mathrm{H}, \mathrm{ArH}), 7.55-7.60\left(\mathrm{~m}, 6 \mathrm{H}, \mathrm{ArH},=\mathrm{CH}-\mathrm{CO},=\mathrm{C} \underline{\mathrm{H}}-\mathrm{OCH}_{3}\right), 7.78(\mathrm{~d}, J=16.0 \mathrm{~Hz}, 1 \mathrm{H},=\mathrm{CH}-\mathrm{Ar}) . \mathrm{EI}$ MS: $m / z(\%) 484\left(\mathrm{M}^{+}, 4\right), 145$ (100), 114 (30), 102 (29), 101 (21). Anal. Calcd for $\mathrm{C}_{31} \mathrm{H}_{32} \mathrm{O}_{5}: \mathrm{C}_{\text {, }}$ 76.84; H, 6.66. Found: C,76.59; H, 6.78.

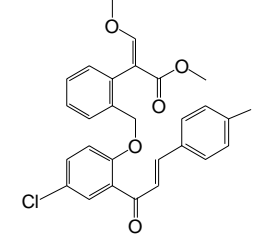

Data for 2a: Yield, 55\%. mp, 68-70 $\square .{ }^{1} \mathrm{H} \mathrm{NMR}\left(400 \mathrm{MHz}, \mathrm{CDCl}_{3}\right): \delta: 2.30\left(\mathrm{~s}, 3 \mathrm{H}, \mathrm{CH}_{3}\right), 3.72(\mathrm{~s}$, $\left.3 \mathrm{H}, \mathrm{COOCH}_{3}\right), 3.84\left(\mathrm{~s}, 3 \mathrm{H}, \mathrm{OCH}_{3}\right), 5.00\left(\mathrm{~s}, 2 \mathrm{H}, \mathrm{CH}_{2}\right), 6.89(\mathrm{~d}, J=9.6 \mathrm{~Hz}, 1 \mathrm{H}, \mathrm{ArH}), 7.11-7.21$ (m, 4H, ArH), 7.27-7.39 (m, 4H, ArH), 7.60-7.64 (m, 4H, ArH, =CH-CO, =C프-OCH 3 ), 7.67 (d, $J$ $=15.2 \mathrm{~Hz}, 1 \mathrm{H},=\mathrm{CH}-\mathrm{Ar}) . \mathrm{MS} \mathrm{m} / \mathrm{z}(\%): 477\left(\mathrm{M}^{+}, 4\right), 206$ (21), 144 (100), 102 (32). Anal. Calcd for $\mathrm{C}_{28} \mathrm{H}_{25} \mathrm{ClO}_{5}$ : C, 70.51; H, 5.28. Found: C, 70.29; H, 5.36.

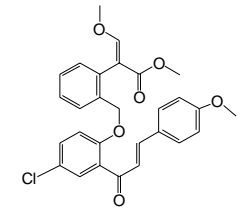

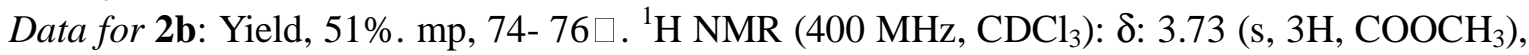
3.84 (s, 3H, $\left.\mathrm{OCH}_{3}\right), 3.98$ (s, 3H, $\left.\mathrm{Ar}-\mathrm{OCH}_{3}\right), 4.99$ (s, 2H, $\mathrm{CH}_{2}$ ), 6.82-6.89 (m, 4H, ArH), 7.18-7.65 $\left(\mathrm{m}, 9 \mathrm{H}, \mathrm{ArH},=\mathrm{CH}-\mathrm{CO},=\mathrm{C} \underline{\mathrm{H}}-\mathrm{OCH}_{3}\right), 7.68(\mathrm{~d}, J=15.2 \mathrm{~Hz}, 1 \mathrm{H},=\mathrm{CH}-\mathrm{Ar}) . \mathrm{MS} m / z(\%): 492\left(\mathrm{M}^{+}\right.$, 3), 146 (26), 144 (100), 131 (27), 102 (36). Anal. Calcd for $\mathrm{C}_{28} \mathrm{H}_{25} \mathrm{ClO}_{6}$ : C, 68.22; $\mathrm{H}, 5.11$. Found: C, 68.39; H, 5.37.

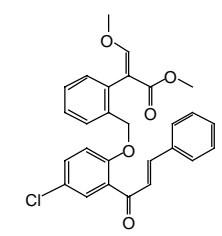

Data for 2c: Yield, 61\%. mp, 53-56 $\square .{ }^{1} \mathrm{H} \mathrm{NMR}\left(400 \mathrm{MHz}, \mathrm{CDCl}_{3}\right): \delta: 3.66\left(\mathrm{~s}, 3 \mathrm{H}, \mathrm{COOCH}_{3}\right)$, $3.74\left(\mathrm{~s}, 3 \mathrm{H}, \mathrm{OCH}_{3}\right), 5.05\left(\mathrm{~s}, 2 \mathrm{H}, \mathrm{CH}_{2}\right), 6.90(\mathrm{~d}, J=8.4 \mathrm{~Hz}, 1 \mathrm{H}, \mathrm{ArH}), 7.18-7.22(\mathrm{~m}, 4 \mathrm{H}, \mathrm{ArH})$, 7.27-7.48 (m, 5H, ArH), 7.52-7.64 (m, 4H, ArH, =CH-CO, =C프-OCH $\left.{ }_{3},=\mathrm{CH}-\mathrm{Ar}\right), 7.70(\mathrm{~d}, J=$ $2.8 \mathrm{~Hz}, 1 \mathrm{H}, \mathrm{ArH})$. EI MS: $m / z$ (\%) $462\left(\mathrm{M}^{+}, 2\right), 205$ (36), 204 (39), 154 (34), 145 (100), 144 (76), 102 (67). Anal. Calcd for $\mathrm{C}_{27} \mathrm{H}_{23} \mathrm{ClO}_{5}$ : C, 57.05; H, 5.01. Found: C, 57.09; H, 5.30.

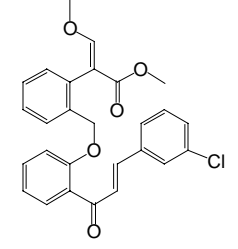

Data for 2d: Yield, 55\%. mp, 58-60 $\square .{ }^{1} \mathrm{H}$ NMR (400 MHz, $\left.\mathrm{CDCl}_{3}\right): \delta: 3.63$ (s, 3H, $\mathrm{COOCH}_{3}$ ), $3.78\left(\mathrm{~s}, 3 \mathrm{H}, \mathrm{OCH}_{3}\right), 5.05\left(\mathrm{~s}, 2 \mathrm{H}, \mathrm{CH}_{2}\right), 7.02$ (d, $\left.J=3.6 \mathrm{~Hz}, 1 \mathrm{H}, \mathrm{ArH}\right), 7.18-7.21(\mathrm{~m}, 4 \mathrm{H}, \mathrm{ArH})$, 7.34-7.41 (m, 4H, ArH), 7.50-7.60 (m, 4H, ArH, =CH-CO, =CH-OCH $7.6 \mathrm{~Hz}, 1 \mathrm{H}, \mathrm{ArH}), 7.98$ (d, J = 1.6 Hz, 1H, ArH). EI MS: m/z (\%) $462\left(\mathrm{M}^{+}, 3\right), 204$ (56), 190 (29), 145 (100), 144 (36), 102 (17). Anal. Calcd for $\mathrm{C}_{27} \mathrm{H}_{23} \mathrm{ClO}_{5}$ : C, 57.05; H, 5.01. Found: C, 57.12; H, 
5.26 .

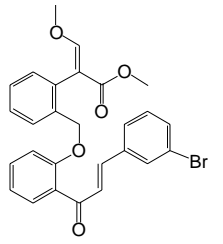

Data for 2e: Yield, 57\%. mp, 71-73 $\square .{ }^{1} \mathrm{H}$ NMR (400 MHz, $\mathrm{CDCl}_{3}$ ): $\delta: 3.79$ (s, 3H, $\mathrm{COOCH}_{3}$ ), $3.86\left(\mathrm{~s}, 3 \mathrm{H}, \mathrm{OCH}_{3}\right), 5.05\left(\mathrm{~s}, 2 \mathrm{H}, \mathrm{CH}_{2}\right), 7.02(\mathrm{~d}, J=4.2 \mathrm{~Hz}, 1 \mathrm{H}, \mathrm{ArH}), 7.18-7.21(\mathrm{~m}, 2 \mathrm{H}, \mathrm{ArH})$, 7.28-7.41 (m, 6H, ArH), 7.50-7.60 (m, 3H, ArH, =CH-CO, =Cㅍ-OCH 3$), 7.68(\mathrm{~d}, J=15.6 \mathrm{~Hz}, 1 \mathrm{H}$, $=\mathrm{CH}-\mathrm{Ar}, 7.98(\mathrm{~d}, J=7.6 \mathrm{~Hz}, 1 \mathrm{H}, \mathrm{ArH}), 8.14(\mathrm{t}, J=1.8 \mathrm{~Hz}, 1 \mathrm{H}, \mathrm{ArH})$. EI MS: $m / z(\%), 507\left(\mathrm{M}^{+}\right.$, 4), 204 (69), 144 (100), 102 (77). Anal. Calcd for $\mathrm{C}_{27} \mathrm{H}_{23} \mathrm{BrO}_{5}$ : C, 63.92; H, 4.57. Found: C, 64.11; $\mathrm{H}, 4.68$.

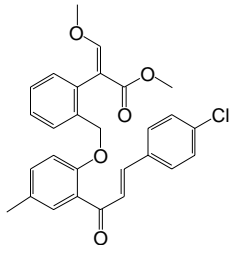

Data for 2f: Yield, 55\%. mp, 53-55 $\square .{ }^{1} \mathrm{H}$ NMR (400 MHz, $\left.\mathrm{CDCl}_{3}\right): \delta: 2.32$ (s, 3H, $\left.\mathrm{CH}_{3}\right), 3.70$ (s, $\left.3 \mathrm{H}, \mathrm{COOCH}_{3}\right), 3.82\left(\mathrm{~s}, 3 \mathrm{H}, \mathrm{OCH}_{3}\right), 5.02\left(\mathrm{~s}, 2 \mathrm{H}, \mathrm{CH}_{2}\right), 6.89$ (d, J=4.4 Hz, 1H, ArH), 7.08-7.23

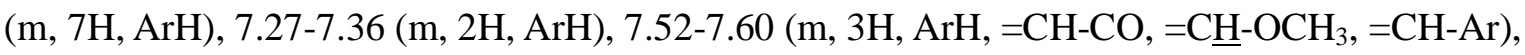
7.67 (d, $J=4.4 \mathrm{~Hz}, 1 \mathrm{H}, \mathrm{ArH})$. MS m/z (\%): $477\left(\mathrm{M}^{+}, 8\right), 205$ (21), 144 (100), 102 (56). Anal. Calcd for $\mathrm{C}_{28} \mathrm{H}_{25} \mathrm{ClO}_{5}$ : C, 70.51; H, 5.28. Found: C, 70.39; H, 5.35.

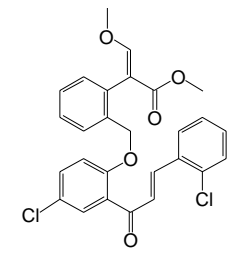

Data for 2g: Yield, 55\%. mp, 59-62 $\square .{ }^{1} \mathrm{H} \mathrm{NMR}\left(400 \mathrm{MHz}, \mathrm{CDCl}_{3}\right): \delta: 3.70\left(\mathrm{~s}, 3 \mathrm{H}, \mathrm{COOCH}_{3}\right)$, $3.82\left(\mathrm{~s}, 3 \mathrm{H}, \mathrm{OCH}_{3}\right), 5.04\left(\mathrm{~s}, 2 \mathrm{H}, \mathrm{CH}_{2}\right), 6.91(\mathrm{~d}, J=8.8 \mathrm{~Hz}, 1 \mathrm{H}, \mathrm{ArH}), 7.09-7.24(\mathrm{~m}, 6 \mathrm{H}, \mathrm{ArH})$, 7.27-7.57 (m, 5H, ArH, =CH-CO, =C $\left.\underline{\mathrm{H}}-\mathrm{OCH}_{3}\right), 7.72(\mathrm{~d}, J=2.4 \mathrm{~Hz}, 1 \mathrm{H}, \mathrm{ArH}), 8.05$ (d, $J=16.0$ $\mathrm{Hz}, 1 \mathrm{H},=\mathrm{CH}-\mathrm{Ar}) . \mathrm{MS} \mathrm{m} / z$ (\%): $497\left(\mathrm{M}^{+}, 11\right), 204$ (100), 176 (39), 114 (94). Anal. Calcd for $\mathrm{C}_{27} \mathrm{H}_{22} \mathrm{Cl}_{2} \mathrm{O}_{5}$ : C, 65.20; H, 4.46. Found: C, 65.48; H, 4.34.

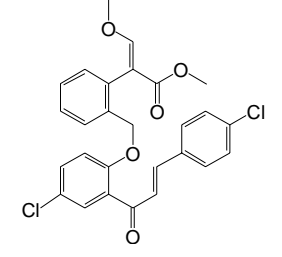

Data for 2h: Yield, 60\%. mp, 68-70 $\left.\square .{ }^{1} \mathrm{H} \mathrm{NMR} \mathrm{(400} \mathrm{MHz,} \mathrm{CDCl}_{3}\right): \delta: 3.68\left(\mathrm{~s}, 3 \mathrm{H}, \mathrm{COOCH}_{3}\right)$, $3.81\left(\mathrm{~s}, 3 \mathrm{H}, \mathrm{OCH}_{3}\right), 5.02\left(\mathrm{~s}, 2 \mathrm{H}, \mathrm{CH}_{2}\right), 6.89$ (d, J = 4.4 Hz, 1H, ArH), 7.08-7.24 (m, 5H, ArH), 7.37-7.51 (m, 7H, ArH, =CH-CO, =C $\left.\underline{\mathrm{H}}-\mathrm{OCH}_{3},=\mathrm{CH}-\mathrm{Ar}\right), 7.59(\mathrm{~d}, J=4.4 \mathrm{~Hz}, 1 \mathrm{H}, \mathrm{ArH}) . \mathrm{MS} \mathrm{m} / z$ (\%): 497 (M+ 3), 204 (100), 177 (51), 114 (64). Anal. Calcd for $\mathrm{C}_{27} \mathrm{H}_{22} \mathrm{Cl}_{2} \mathrm{O}_{5}: \mathrm{C}, 65.20 ; \mathrm{H}, 4.46$. Found: C, 65.37; H, 4.13. 


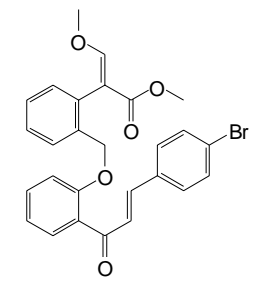

Data for 2i: Yield, 55\%. mp, 63-64 $\square .{ }^{1} \mathrm{H} \mathrm{NMR}\left(400 \mathrm{MHz}, \mathrm{CDCl}_{3}\right): \delta: 3.70\left(\mathrm{~s}, 3 \mathrm{H}, \mathrm{COOCH}_{3}\right)$, $3.82\left(\mathrm{~s}, 3 \mathrm{H}, \mathrm{OCH}_{3}\right), 5.04\left(\mathrm{~s}, 2 \mathrm{H}, \mathrm{CH}_{2}\right), 7.14-7.21(\mathrm{~m}, 2 \mathrm{H}, \mathrm{ArH}), 7.34-7.43(\mathrm{~m}, 6 \mathrm{H}, \mathrm{ArH})$, 7.47-7.60 (m, 6H, ArH, =CH-CO, $\left.=\mathrm{CH}-\mathrm{OCH}_{3},=\mathrm{CH}-\mathrm{Ar}\right), 7.74(\mathrm{~d}, J=4.8 \mathrm{~Hz}, 1 \mathrm{H}, \mathrm{ArH}) . \mathrm{MS} \mathrm{m} / z$ (\%): 507 (M+2), 204 (21), 146 (44), 145 (100), 144 (93), 114 (46), 102(42). Anal. Calcd for $\mathrm{C}_{27} \mathrm{H}_{23} \mathrm{BrO}_{5}$ : C, 63.92; H, 4.57. Found: C, 64.05; H, 4.59.

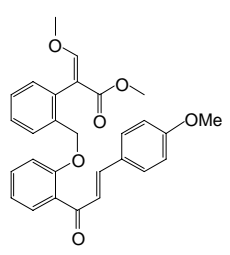

Data for 2j: Yield, 55\%. mp, 59-62 $\square .{ }^{1} \mathrm{H} \mathrm{NMR}\left(400 \mathrm{MHz}, \mathrm{CDCl}_{3}\right.$ ): $\delta: 3.70$ (s, 3H, $\mathrm{COOCH}_{3}$ ), $3.82\left(\mathrm{~s}, 3 \mathrm{H}, \mathrm{OCH}_{3}\right), 3.98\left(\mathrm{~s}, 3 \mathrm{H}, \mathrm{ArOCH}_{3}\right), 5.04\left(\mathrm{~s}, 2 \mathrm{H}, \mathrm{CH}_{2}\right), 6.82(\mathrm{~d}, J=8.8 \mathrm{~Hz}, 2 \mathrm{H}, \operatorname{ArH})$, 6.94-7.04 (m, 2H, ArH), 7.18-7.22 (m, 2H, ArH), 7.31-7.47 (m, 5H, ArH), 7.60-7.65 (m, 3H, ArH, $\left.=\mathrm{CH}-\mathrm{CO},=\mathrm{C} \underline{\mathrm{H}}-\mathrm{OCH}_{3},=\mathrm{CH}-\mathrm{Ar}\right), 7.73(\mathrm{~d}, J=7.6 \mathrm{~Hz}, 1 \mathrm{H}, \mathrm{ArH}) . \mathrm{MS} m / z(\%): 458\left(\mathrm{M}^{+}, 14\right), 426$ $\left(\mathrm{M}^{+}, 24\right), 36$ (27), 250 (96), 204 (100), 114(67), 102 (72). Anal. Calcd for $\mathrm{C}_{28} \mathrm{H}_{26} \mathrm{O}_{6}: \mathrm{C}, 73.35 ; \mathrm{H}$, 5.72. Found: C, 73.62; H, 5.80.

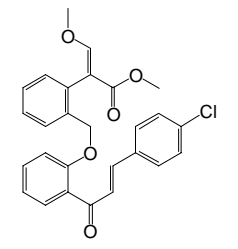

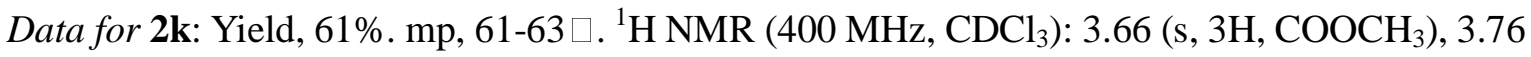
(s, 3H, $\left.\mathrm{OCH}_{3}\right), 5.05\left(\mathrm{~s}, 2 \mathrm{H}, \mathrm{CH}_{2}\right), 7.02-7.09$ (m, 3H, $\left.\mathrm{ArH}\right), 7.15-7.47(\mathrm{~m}, 8 \mathrm{H}, \mathrm{ArH}), 7.51-7.61(\mathrm{~m}$, $\left.3 \mathrm{H}, \mathrm{ArH},=\mathrm{CH}-\mathrm{CO},=\mathrm{C} \underline{\mathrm{H}}-\mathrm{OCH}_{3},=\mathrm{CH}-\mathrm{Ar}\right), 7.79(\mathrm{~d}, J=6.0 \mathrm{~Hz}, 1 \mathrm{H}, \mathrm{ArH}) . \mathrm{EI} \mathrm{MS}: m / z(\%) 462$ $\left(\mathrm{M}^{+}, 4\right), 204$ (29), 145 (100), 102 (29). Anal. Calcd for $\mathrm{C}_{27} \mathrm{H}_{23} \mathrm{ClO}_{5}$ : C, 57.05; H, 5.01. Found: C, 57.35; H, 5.21 .

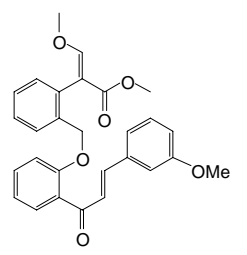

Data for 2l: Yield, 55\%. mp, 66-69 $\square .{ }^{1} \mathrm{H}$ NMR (400 MHz, $\mathrm{CDCl}_{3}$ ): $\delta: 3.70$ (s, 3H, $\mathrm{COOCH}_{3}$ ), $3.81\left(\mathrm{~s}, 3 \mathrm{H}, \mathrm{OCH}_{3}\right), 3.96$ (s, 3H, Ar- $\left.\mathrm{OCH}_{3}\right), 5.04\left(\mathrm{~s}, 2 \mathrm{H}, \mathrm{CH}_{2}\right), 6.94-7.05$ (m, 4H, ArH), 7.15-7.43 $(\mathrm{m}, 6 \mathrm{H}, \mathrm{ArH}), 7.51-7.62\left(\mathrm{~m}, 4 \mathrm{H}, \mathrm{ArH},=\mathrm{CH}-\mathrm{CO},=\mathrm{CH}_{-}-\mathrm{OCH}_{3},=\mathrm{CH}-\mathrm{Ar}\right), 7.73(\mathrm{~d}, J=7.6 \mathrm{~Hz}, 1 \mathrm{H}$, $=\mathrm{CH}-\mathrm{Ar}) . \mathrm{MS} \mathrm{m} / z(\%): 458\left(\mathrm{M}^{+}, 17\right), 426\left(\mathrm{M}^{+}, 34\right), 204$ (100), 114 (87), 102 (54). Anal. Calcd for $\mathrm{C}_{28} \mathrm{H}_{26} \mathrm{O}_{6}$ : C, 73.35; H, 5.72. Found: C, 73.64, H, 5.51. 


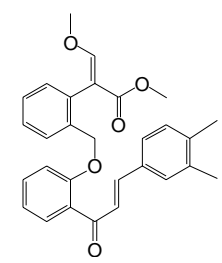

Data for 2m: Yield, 53\%. mp, 56-58 $\square .{ }^{1} \mathrm{H}$ NMR (400 MHz, $\left.\mathrm{CDCl}_{3}\right): \delta: 2.22\left(\mathrm{~s}, 3 \mathrm{H}, \mathrm{CH}_{3}\right), 2.27(\mathrm{~s}$, $\left.3 \mathrm{H}, \mathrm{CH}_{3}\right), 3.67\left(\mathrm{~s}, 3 \mathrm{H}, \mathrm{COOCH}_{3}\right), 3.78\left(\mathrm{~s}, 3 \mathrm{H}, \mathrm{OCH}_{3}\right), 5.06\left(\mathrm{~s}, 2 \mathrm{H}, \mathrm{CH}_{2}\right), 6.93(\mathrm{~d}, J=8.0 \mathrm{~Hz}, 1 \mathrm{H}$, ArH ), 7.02-7.08 (m, 3H, ArH), 7.13-7.30 (m, 4H, ArH), 7.40-7.57 (m, 3H, ArH, =CH-CO, $\left.=\mathrm{CH}_{-}-\mathrm{OCH}_{3}\right), 7.61-7.71(\mathrm{~m}, 3 \mathrm{H}, \mathrm{ArH},=\mathrm{CH}-\mathrm{Ar}) . \mathrm{EI} \mathrm{MS:} \mathrm{m} / z(\%) 456\left(\mathrm{M}^{+}, 8\right), 205$ (38), 144 (100), 114 (24), 102 (33). Anal. Calcd for $\mathrm{C}_{29} \mathrm{H}_{28} \mathrm{O}_{5}$ : C, 76.30; H, 6.18 . Found: C, 76.56; H, 6.11.

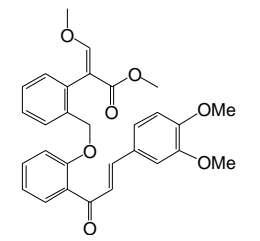

Data for 2n: Yield, 55\%. mp, 93-95 $\square .{ }^{1} \mathrm{H} \mathrm{NMR}\left(400 \mathrm{MHz}, \mathrm{CDCl}_{3}\right.$ ): $\delta: 3.67$ (s, $3 \mathrm{H}, \mathrm{COOCH}_{3}$ ), $3.80\left(\mathrm{~s}, 3 \mathrm{H}, \mathrm{OCH}_{3}\right), 3.89\left(\mathrm{~s}, 3 \mathrm{H}, \mathrm{Ar}-\mathrm{OCH}_{3}\right), 3.91\left(\mathrm{~s}, 3 \mathrm{H}, \mathrm{Ar}-\mathrm{OCH}_{3}\right), 5.06\left(\mathrm{~s}, 2 \mathrm{H}, \mathrm{CH}_{2}\right), 6.90(\mathrm{~d}, J$ $=8.8 \mathrm{~Hz}, 1 \mathrm{H}, \mathrm{ArH}), 7.16-7.27$ (m, 4H, ArH), 7.31-7.37 (m, 3H, ArH), 7.40-7.44 (m, 2H, ArH), 7.58-7.72 (m, 4H, ArH, $\left.=\mathrm{CH}-\mathrm{CO},=\mathrm{CH}-\mathrm{OCH}_{3},=\mathrm{CH}-\mathrm{Ar}\right) . \mathrm{EI} \mathrm{MS:} m / z(\%) 488\left(\mathrm{M}^{+}, 32\right), 314(25)$, 205 (31), 144 (100), 102 (65). Anal. Calcd for $\mathrm{C}_{29} \mathrm{H}_{28} \mathrm{O}_{7}$ : C, 71.30; H, 5.78 . Found: C, 71.59; H, 6.04 .

CP01

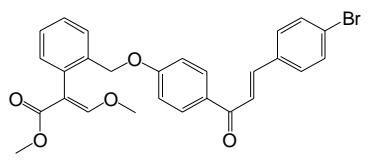

3a: Yield, 81\%. mp, 132-134 $\square .{ }^{1} \mathrm{H}$ NMR (400 MHz, $\mathrm{CDCl}_{3}$ ): $\delta: 3.68$ (s, 3H, $\left.\mathrm{COOCH}_{3}\right), 3.81$ (s, $\left.3 \mathrm{H}, \mathrm{OCH}_{3}\right), 5.06\left(\mathrm{~s}, 2 \mathrm{H}, \mathrm{CH}_{2}\right), 6.98(\mathrm{~d}, J=8.8 \mathrm{~Hz}, 2 \mathrm{H}, \mathrm{ArH}), 7.20-7.36(\mathrm{~m}, 3 \mathrm{H}, \mathrm{ArH}), 7.49-7.56$ $(\mathrm{m}, 6 \mathrm{H}, \mathrm{ArH},=\mathrm{CH}-\mathrm{CO}), 7.61\left(\mathrm{~s}, 1 \mathrm{H},=\mathrm{CH}-\mathrm{OCH}_{3}\right), 7.72(\mathrm{~d}, J=15.6 \mathrm{~Hz}, 1 \mathrm{H},=\mathrm{CH}-\mathrm{Ar}), 8.01(\mathrm{~d}, J$ $=8.4 \mathrm{~Hz}, 2 \mathrm{H}, \mathrm{ArH})$. EI MS: $m / z(\%) 508\left([\mathrm{M}+1]^{+}, 9\right), 507\left(\mathrm{M}^{+}, 9\right), 304$ (30), 303 (29), 302 (32), 300 (38), 222 (48), 204 (97), 193 (37), 176 (65), 173 (94), 165 (92), 144 (99), 129 (100), 120 (51), 116 (22), 102 (96). Anal. Calcd for $\mathrm{C}_{27} \mathrm{H}_{23} \mathrm{BrO}_{5}$ : C, 63.92; H, 4.57. Found: C, 63.99; H, 4.30

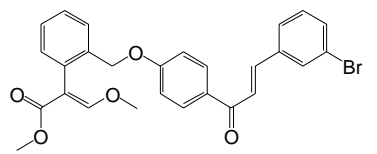

3b: Yield, 77\%. mp, 123-124 $\square .{ }^{1} \mathrm{H}$ NMR (400 MHz, $\mathrm{CDCl}_{3}$ ): $\delta: 3.72$ (s, 3H, $\left.\mathrm{COOCH}_{3}\right), 3.85$ (s, $\left.3 \mathrm{H}, \mathrm{OCH}_{3}\right), 5.06\left(\mathrm{~s}, 2 \mathrm{H}, \mathrm{CH}_{2}\right), 6.98(\mathrm{~d}, J=8.4 \mathrm{~Hz}, 2 \mathrm{H}, \mathrm{ArH}), 7.20-7.37$ (m, 4H, ArH), 7.50-7.54 $(\mathrm{m}, 4 \mathrm{H}, \mathrm{ArH},=\mathrm{CH}-\mathrm{CO}), 7.62\left(\mathrm{~s}, 1 \mathrm{H},=\mathrm{CH}-\mathrm{OCH}_{3}\right), 7.73(\mathrm{~d}, J=16.4 \mathrm{~Hz}, 1 \mathrm{H},=\mathrm{CH}-\mathrm{Ar}), 7.79(\mathrm{~s}, 1 \mathrm{H}$, ArH), $8.02(\mathrm{~d}, J=8.4 \mathrm{~Hz}, 2 \mathrm{H}, \mathrm{ArH})$. EI MS: $m / z(\%) 508$ ([M+1] $\left.]^{+}, 7\right), 507\left(\mathrm{M}^{+}, 4\right), 304(27), 303$ (26), 302 (32), 300 (31), 223 (26), 222 (22), 204 (98), 194 (24), 176 (66), 173 (96), 165 (65), 144 (100), 129 (98), 120 (55), 117 (32), 114 (77), 102 (98). Anal. Calcd for $\mathrm{C}_{27} \mathrm{H}_{23} \mathrm{BrO}_{5}$ : C, 63.92; $\mathrm{H}$, 4.57. Found: C, 64.12; H, 4.62.

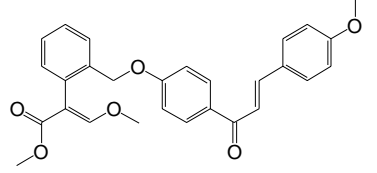


3c: Yield, 79\%. mp, 171-173 $\square .{ }^{1} \mathrm{H}$ NMR (400 MHz, $\left.\mathrm{CDCl}_{3}\right): \delta: 3.72$ (s, 3H, $\mathrm{COOCH}_{3}$ ), 3.84 (s, $\left.3 \mathrm{H}, \mathrm{OCH}_{3}\right), 3.86\left(\mathrm{~s}, 3 \mathrm{H}, \mathrm{Ar}-\mathrm{OCH}_{3}\right), 5.06\left(\mathrm{~s}, 2 \mathrm{H}, \mathrm{CH}_{2}\right), 6.93-6.99(\mathrm{~m}, 4 \mathrm{H}, \mathrm{ArH}), 7.20-7.21(\mathrm{~m}, 1 \mathrm{H}$, $\mathrm{ArH}), 7.34-7.44(\mathrm{~m}, 3 \mathrm{H}, \mathrm{ArH}), 7.51-7.61\left(\mathrm{~m}, 4 \mathrm{H}, \mathrm{ArH},=\mathrm{CH}-\mathrm{CO},=\mathrm{CH}-\mathrm{OCH}_{3}\right), 7.76(\mathrm{~d}, J=$ 15.6Hz, 1H, =CH-Ar), 8.01 (d, $J=8.4 \mathrm{~Hz}, 2 \mathrm{H}, \mathrm{ArH})$. EI MS: $m / z$ (\%) $458\left(\mathrm{M}^{+}, 1\right), 224$ (7), 204 (65), 176 (7), 172 (8), 146 (13), 144 (100), 131 (8), 114 (7). Anal. Calcd for $\mathrm{C}_{28} \mathrm{H}_{26} \mathrm{O}_{6}$ : C, 73.35 ; H, 5.72. Found: C, 73.19; H, 5.65.

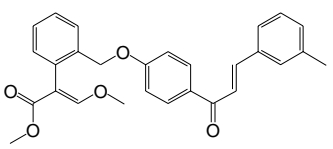

3d: Yield, 83\%. mp, 158-160 $\square .{ }^{1} \mathrm{H}$ NMR (400 MHz, $\left.\mathrm{CDCl}_{3}\right): \delta: 2.40\left(\mathrm{~s}, 3 \mathrm{H}, \mathrm{CH}_{3}\right), 3.72(\mathrm{~s}, 3 \mathrm{H}$, $\left.\mathrm{COOCH}_{3}\right), 3.84\left(\mathrm{~s}, 3 \mathrm{H}, \mathrm{OCH}_{3}\right), 5.06\left(\mathrm{~s}, 2 \mathrm{H}, \mathrm{CH}_{2}\right), 6.98(\mathrm{~d}, J=7.6 \mathrm{~Hz}, 2 \mathrm{H}, \mathrm{ArH}), 7.21-7.61(\mathrm{~m}$, $\left.10 \mathrm{H}, \mathrm{ArH},=\mathrm{CH}-\mathrm{CO},=\mathrm{C} \underline{\mathrm{H}}-\mathrm{OCH}_{3}\right), 7.78(\mathrm{~d}, J=15.6 \mathrm{~Hz}, 1 \mathrm{H},=\mathrm{CH}-\mathrm{Ar}), 8.01(\mathrm{~d}, J=8.8 \mathrm{~Hz}, 2 \mathrm{H}$, ArH). EI MS: $m / z(\%) 443\left([\mathrm{M}+1]^{+}, 5\right), 442\left(\mathrm{M}^{+}, 19\right), 237$ (35), 222 (40), 204 (99), 177 (37), 173 (45), 165 (41), 144 (100), 131 (67), 129 (25), 127 (20), 114 (67), 103 (34), 102 (38). Anal. Calcd for $\mathrm{C}_{28} \mathrm{H}_{26} \mathrm{O}_{5}$ : C, 76.00; H, 5.92. Found: C, 76.23; H, 5.83.

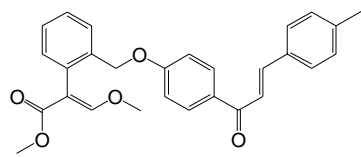

3e: Yield, 78\%. mp, 164-165 $\square .{ }^{1} \mathrm{H}$ NMR (400 MHz, $\mathrm{CDCl}_{3}$ ): $\delta: 2.39$ (s, 3H, $\left.\mathrm{CH}_{3}\right), 3.72(\mathrm{~s}, 3 \mathrm{H}$, $\left.\mathrm{COOCH}_{3}\right), 3.84\left(\mathrm{~s}, 3 \mathrm{H}, \mathrm{OCH}_{3}\right), 5.06\left(\mathrm{~s}, 2 \mathrm{H}, \mathrm{CH}_{2}\right), 6.98(\mathrm{~d}, J=9.2 \mathrm{~Hz}, 2 \mathrm{H}, \mathrm{ArH}), 7.21-7.23(\mathrm{~m}, 3 \mathrm{H}$, ArH), 7.34-7.36 (m, 2H, ArH), 7.47-7.55(m, 4H, ArH, =CH-CO), 7.61 (s, 1H, =CH-OCH $)_{3}, 7.76$ $(\mathrm{d}, J=15.6 \mathrm{~Hz}, 1 \mathrm{H},=\mathrm{CH}-\mathrm{Ar}), 8.01(\mathrm{~d}, J=8.8 \mathrm{~Hz}, 2 \mathrm{H}, \mathrm{ArH})$. EI MS: $m / z(\%) 443\left([\mathrm{M}+1]^{+}, 4\right), 442$ $\left(\mathrm{M}^{+}, 11\right), 238$ (20), 236 (40), 204 (99), 177 (24), 173 (42), 165 (33), 144 (100), 131 (36), 130 (23), 114 (60), 103 (30), 102 (27). Anal. Calcd for $\mathrm{C}_{28} \mathrm{H}_{26} \mathrm{O}_{5}$ : C, 76.00; H, 5.92. Found: C, 76.11; H, 6.22 .

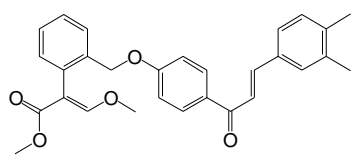

3f: Yield, 82\%. mp, 124-126 $\square .{ }^{1} \mathrm{H}$ NMR (400 MHz, $\mathrm{CDCl}_{3}$ ): $\delta: 2.30$ (s, 6H, 2× $\mathrm{ArCH}_{3}$ ), 3.72 (s, $\left.3 \mathrm{H}, \mathrm{COOCH}_{3}\right), 3.84\left(\mathrm{~s}, 3 \mathrm{H}, \mathrm{OCH}_{3}\right), 5.06\left(\mathrm{~s}, 2 \mathrm{H}, \mathrm{CH}_{2}\right), 6.96(\mathrm{~d}, J=8.8 \mathrm{~Hz}, 2 \mathrm{H}, \mathrm{ArH}), 7.18-7.50$ $(\mathrm{m}, 8 \mathrm{H}, \mathrm{ArH},=\mathrm{CH}-\mathrm{CO}), 7.60\left(\mathrm{~s}, 1 \mathrm{H},=\mathrm{CH}-\mathrm{OCH}_{3}\right), 7.74(\mathrm{~d}, J=15.6 \mathrm{~Hz}, 1 \mathrm{H},=\mathrm{CH}-\mathrm{Ar}), 8.01(\mathrm{~d}, J$ $=9.2 \mathrm{~Hz}, 2 \mathrm{H}, \mathrm{ArH}) . \mathrm{EI} \mathrm{MS:} m / z(\%) 457\left([\mathrm{M}+1]^{+}, 11\right), 456\left(\mathrm{M}^{+}, 28\right), 252$ (30), 251 (61), $236(94)$, 222 (28), 208 (26), 204 (98), 177 (67), 173 (97), 165 (51), 158 (65), 144 (100), 128 (99), 114 (98), 105 (18). Anal. Calcd for $\mathrm{C}_{29} \mathrm{H}_{28} \mathrm{O}_{5}$ : C, 76.30; H, 6.18 . Found: C, 76.01; H,6.35.

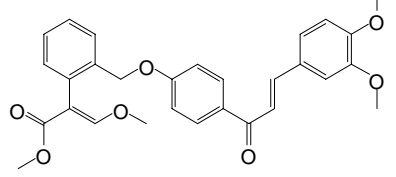

3g: Yield, 78\%. mp, 184-186 $\square .{ }^{1} \mathrm{H}$ NMR (400 MHz, $\mathrm{CDCl}_{3}$ ): $\delta: 3.72$ (s, 3H, $\mathrm{COOCH}_{3}$ ), 3.84 (s, $\left.3 \mathrm{H}, \mathrm{OCH}_{3}\right), 3.93\left(\mathrm{~s}, 3 \mathrm{H}, \mathrm{Ar}-\mathrm{OCH}_{3}\right), 3.95\left(\mathrm{~s}, 3 \mathrm{H}, \mathrm{Ar}-\mathrm{OCH}_{3}\right), 5.06\left(\mathrm{~s}, 2 \mathrm{H}, \mathrm{CH}_{2}\right), 6.88-6.99(\mathrm{~m}, 4 \mathrm{H}$, $\mathrm{ArH})$, 7.15-7.26 (m, 3H, ArH)), 7.34-7.56 (m, 3H, ArH, =CH-CO), $7.60\left(\mathrm{~s}, 1 \mathrm{H},=\mathrm{CH}-\mathrm{OCH}_{3}\right)$, $7.74(\mathrm{~d}, J=15.6 \mathrm{~Hz}, 1 \mathrm{H},=\mathrm{CH}-\mathrm{Ar}), 8.01(\mathrm{~d}, J=8.8 \mathrm{~Hz}, 2 \mathrm{H}, \mathrm{ArH})$. EI MS: $m / z(\%) 489\left([\mathrm{M}+1]^{+}, 4\right)$, 488 (M+19), 204 (74), 173 (12), 146 (28), 144 (100), 131 (18), 130 (10), 114 (20), 103 (13), 102 
(11). Anal. Calcd for $\mathrm{C}_{30} \mathrm{H}_{32} \mathrm{O}_{7}$ : C,71.30; H, 5.78 . Found: C, 71.46; H,5.91.

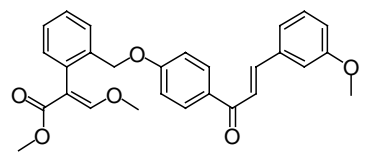

3h: Yield, 75\%. mp, 104-106.${ }^{1} \mathrm{H}$ NMR (400 MHz, $\left.\mathrm{CDCl}_{3}\right): \delta: 3.71$ (s, 3H, $\left.\mathrm{COOCH}_{3}\right), 3.84$ (s, $\left.3 \mathrm{H}, \mathrm{OCH}_{3}\right), 3.93\left(\mathrm{~s}, 3 \mathrm{H}, \mathrm{Ar}-\mathrm{OCH}_{3}\right), 5.06\left(\mathrm{~s}, 2 \mathrm{H}, \mathrm{CH}_{2}\right), 6.95-6.99(\mathrm{~m}, 3 \mathrm{H}, \mathrm{ArH}), 7.15-7.25(\mathrm{~m}, 3 \mathrm{H}$, $\mathrm{ArH}), 7.31-7.36(\mathrm{~m}, 3 \mathrm{H}, \mathrm{ArH}), 7.49-7.53(\mathrm{~m}, 2 \mathrm{H}, \mathrm{ArH},=\mathrm{CH}-\mathrm{CO}), 7.60\left(\mathrm{~s}, 1 \mathrm{H},=\mathrm{CH}-\mathrm{OCH}_{3}\right), 7.76$ $(\mathrm{d}, J=15.6 \mathrm{~Hz}, 1 \mathrm{H},=\mathrm{CH}-\mathrm{Ar}), 8.00(\mathrm{~d}, J=8.8 \mathrm{~Hz}, 2 \mathrm{H}, \mathrm{ArH})$. EI MS: $m / z(\%) 458\left(\mathrm{M}^{+}, 5\right), 252(10)$, 204 (87), 177 (11), 146 (26), 144 (100), 131 (20), 130 (10), 114 (22), 103 (18), 102 (16). Anal Calcd for $\mathrm{C}_{28} \mathrm{H}_{26} \mathrm{O}_{6}$ : C, 73.35; H, 5.72. Found: C, 73.07; H, 5.75.

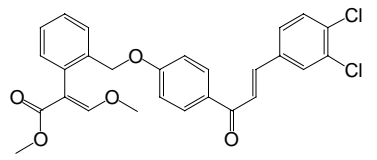

3i: Yield, 78\%. mp, 134-136 $\square .1 \mathrm{H} \mathrm{NMR} \mathrm{(400} \mathrm{MHz,} \mathrm{CDCl}_{3}$ ): $\delta: 3.72$ (s, 3H, $\left.\mathrm{COOCH}_{3}\right), 3.84$ (s, $\left.3 \mathrm{H}, \mathrm{OCH}_{3}\right), 5.06\left(\mathrm{~s}, 2 \mathrm{H}, \mathrm{CH}_{2}\right), 6.97(\mathrm{~d}, J=8.8 \mathrm{~Hz}, 2 \mathrm{H}, \mathrm{ArH}), 7.23-7.51(\mathrm{~m}, 8 \mathrm{H}, \mathrm{ArH}), 7.60(\mathrm{~s}, 1 \mathrm{H}$, $\left.=\mathrm{CH}-\mathrm{OCH}_{3}\right), 7.71(\mathrm{~d}, J=1.2 \mathrm{~Hz}, 1 \mathrm{H},=\mathrm{CH}-\mathrm{CO}), 7.97-7.99(\mathrm{~m}, 2 \mathrm{H}, \mathrm{ArH},=\mathrm{CH}-\mathrm{Ar}) . \mathrm{EI} \mathrm{MS}: \mathrm{m} / \mathrm{z}$ (\%) 497 (M+, 2), 206 (11), 204 (86), 177 (10), 173 (12), 146 (43), 144 (100), 131 (22), 114 (19), 103 (19). Anal. Calcd for $\mathrm{C}_{27} \mathrm{H}_{22} \mathrm{Cl}_{2} \mathrm{O}_{5}$ : C, 65.20; H, 4.46. Found: C, 65.46; H, 4.26.

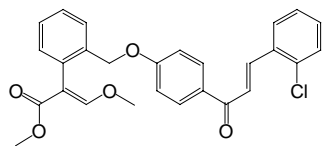

3j: Yield, 80\%. mp, 68-70 $\square .{ }^{1} \mathrm{H}$ NMR (400 MHz, $\mathrm{CDCl}_{3}$ ): $\delta: 3.71\left(\mathrm{~s}, 3 \mathrm{H}, \mathrm{COOCH}_{3}\right), 3.83(\mathrm{~s}, 3 \mathrm{H}$, $\left.\mathrm{OCH}_{3}\right), 5.06\left(\mathrm{~s}, 2 \mathrm{H}, \mathrm{CH}_{2}\right), 6.99(\mathrm{~d}, J=8.8 \mathrm{~Hz}, 2 \mathrm{H}, \mathrm{ArH}), 7.20-7.36(\mathrm{~m}, 6 \mathrm{H}, \mathrm{ArH}), 7.43-7.51(\mathrm{~m}$, $2 \mathrm{H}, \mathrm{ArH}), 7.60\left(\mathrm{~s}, 1 \mathrm{H},=\mathrm{CH}-\mathrm{OCH}_{3}\right), 7.76(\mathrm{~d}, J=4.2 \mathrm{~Hz}, 1 \mathrm{H},=\mathrm{CH}-\mathrm{CO}), 8.00(\mathrm{~d}, J=4.4 \mathrm{~Hz}, 2 \mathrm{H}$, $\mathrm{ArH}), 8.15(\mathrm{~d}, J=15.6 \mathrm{~Hz}, 1 \mathrm{H},=\mathrm{CH}-\mathrm{Ar})$. EI MS: $m / z(\%) 463\left([\mathrm{M}+1]^{+}, 9\right), 462\left(\mathrm{M}^{+}, 20\right), 223(46)$, 206 (32), 204 (100), 193 (40), 177 (32), 165 (63), 146 (64), 145 (91), 131 (57), 114 (60), 103 (58), 101 (42). Anal. Calcd for $\mathrm{C}_{27} \mathrm{H}_{23} \mathrm{ClO}_{5}$ : C, 70.05; H, 5.01. Found: C, 69.86; H, 5.11.

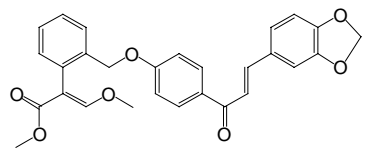

3k: Yield, 81\%. mp, 181-183 $\square .{ }^{1} \mathrm{H}$ NMR (400 MHz, $\mathrm{CDCl}_{3}$ ): $\delta: 3.71$ (s, 3H, $\mathrm{COOCH}_{3}$ ), 3.83 (s, $\left.3 \mathrm{H}, \mathrm{OCH}_{3}\right), 5.05\left(\mathrm{~s}, 2 \mathrm{H}, \mathrm{CH}_{2}\right), 6.02\left(\mathrm{~s}, 2 \mathrm{H},-\mathrm{OCH}_{2} \mathrm{O}-\right), 6.84(\mathrm{~d}, J=8.4 \mathrm{~Hz}, 1 \mathrm{H}, \mathrm{ArH}), 6.98(\mathrm{~d}, J=$ $8.8 \mathrm{~Hz}, 2 \mathrm{H}, \mathrm{ArH}), 7.15-7.24(\mathrm{~m}, 3 \mathrm{H}, \mathrm{ArH}), 7.33-7.56(\mathrm{~m}, 4 \mathrm{H}, \mathrm{ArH},=\mathrm{CH}-\mathrm{CO}), 7.60(\mathrm{~s}, 1 \mathrm{H}$, $\left.=\mathrm{CH}-\mathrm{OCH}_{3}\right), 7.69(\mathrm{~d}, J=8.0 \mathrm{~Hz}, 2 \mathrm{H}, \mathrm{ArH}), 7.99(\mathrm{~d}, J=15.6 \mathrm{~Hz}, 1 \mathrm{H},=\mathrm{CH}-\mathrm{Ar})$. EI MS: $m / z(\%)$ $473\left([\mathrm{M}+1]^{+}, 4\right), 472\left(\mathrm{M}^{+}, 18\right), 268$ (22), 266 (18), 206 (17), 204 (88), 181 (18), 177 (17), 172 (30), 144 (100), 131 (29), 130 (21), 114 (39). Anal. Calcd for $\mathrm{C}_{28} \mathrm{H}_{24} \mathrm{O}_{7}$ : C, 71.18; H, 5.12; Found: C, 71.21; H, 5.02.

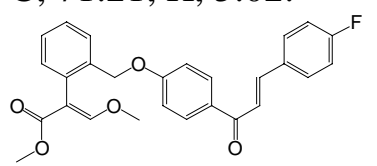

31: Yield, 77\%. mp, 128-130 $\square .1 \mathrm{H} \mathrm{NMR} \mathrm{(400} \mathrm{MHz,} \mathrm{CDCl}_{3}$ ): $\delta: 3.72$ (s, 3H, $\left.\mathrm{COOCH}_{3}\right), 3.84$ (s, $\left.3 \mathrm{H}, \mathrm{OCH}_{3}\right), 5.06\left(\mathrm{~s}, 2 \mathrm{H}, \mathrm{CH}_{2}\right), 6.99(\mathrm{~d}, J=4.4 \mathrm{~Hz}, 2 \mathrm{H}, \mathrm{ArH}), 7.10-7.36(\mathrm{~m}, 4 \mathrm{H}, \mathrm{ArH}), 7.43-7.47$ 
$(\mathrm{m}, 3 \mathrm{H}, \mathrm{ArH},=\mathrm{CH}-\mathrm{CO}), 7.60-7.64\left(\mathrm{~m}, 3 \mathrm{H}, \mathrm{ArH},=\mathrm{CH}-\mathrm{OCH}_{3}\right), 7.75(\mathrm{~d}, J=15.6 \mathrm{~Hz}, 1 \mathrm{H},=\mathrm{CH}-\mathrm{Ar})$, $8.00(\mathrm{~d}, J=8.8 \mathrm{~Hz}, 2 \mathrm{H}, \mathrm{ArH})$. EI MS: $m / z(\%) 446\left(\mathrm{M}^{+}, 3\right), 204$ (62), 165 (10), 163 (23), 149 (10), 146 (25), 145 (100), 131 (21), 121 (15), 114 (15). Anal. Calcd for $\mathrm{C}_{27} \mathrm{H}_{23} \mathrm{FO}_{5}$ : C, 72.63; H, 5.19. Found: C,72.77; H, 5.40.

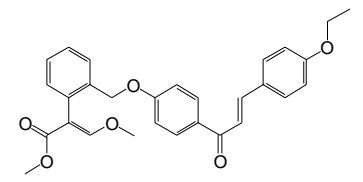

3m: Yield, 74\%. mp, 181-183 $\square .{ }^{1} \mathrm{H}$ NMR (400 MHz, $\mathrm{CDCl}_{3}$ ): $\delta: 1.43$ (t, $J=7.6 \mathrm{~Hz}, 3 \mathrm{H}, \mathrm{CH}_{3}$ ), $3.70\left(\mathrm{~s}, 3 \mathrm{H}, \mathrm{COOCH}_{3}\right), 3.83\left(\mathrm{~s}, 3 \mathrm{H}, \mathrm{OCH}_{3}\right), 4.08\left(\mathrm{dd}, J_{1}=7.2 \mathrm{~Hz}, J_{2}=13.2 \mathrm{~Hz}, 2 \mathrm{H}, \mathrm{CH}_{2} \mathrm{CH}_{3}\right), 5.04$ (s, 2H, $\mathrm{CH}_{2}$ ), 6.90-6.98 (m, 4H, ArH), 7.20-7.42 (m, 5H, ArH), 7.56-7.60 (m, 3H, ArH, =CH-CO, $\left.=\mathrm{C}^{\mathrm{H}}-\mathrm{OCH}_{3}\right), 7.76(\mathrm{~d}, J=15.6 \mathrm{~Hz}, 1 \mathrm{H},=\mathrm{CH}-\mathrm{Ar}), 7.99(\mathrm{~d}, J=8.8 \mathrm{~Hz}, 2 \mathrm{H}, \mathrm{ArH}) . \mathrm{EI} \mathrm{MS}: \mathrm{m} / z(\%)$ $473\left([\mathrm{M}+1]^{+}, 5\right), 472\left(\mathrm{M}^{+}, 15\right), 204$ (44), 165 (28), 145 (70), 144 (100), 130 (40), 121 (23), 114 (39), 103 (65), 102 (55). Anal. Calcd for $\mathrm{C}_{29} \mathrm{H}_{28} \mathrm{O}_{6}$ : C, 73.71; H, 5.97. Found: C, 73.79; H, 6.13.

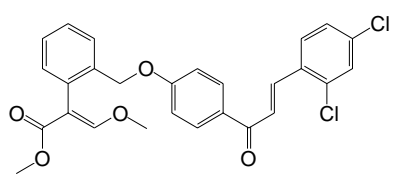

3n: Yield, 84\%. mp, 59-61 $\square .{ }^{1} \mathrm{H}$ NMR (400 MHz, $\mathrm{CDCl}_{3}$ ): $\delta: 3.71$ (s, 3H, $\mathrm{COOCH}_{3}$ ), 3.83 (s, 3H, $\left.\mathrm{OCH}_{3}\right), 5.06\left(\mathrm{~s}, 2 \mathrm{H}, \mathrm{CH}_{2}\right), 6.99(\mathrm{~d}, J=8.8 \mathrm{~Hz}, 2 \mathrm{H}, \mathrm{ArH}), 7.20-7.36(\mathrm{~m}, 4 \mathrm{H}, \mathrm{ArH}), 7.44-7.48(\mathrm{~m}$, $3 \mathrm{H}, \mathrm{ArH}), 7.61\left(\mathrm{~s}, 1 \mathrm{H},=\mathrm{CH}-\mathrm{OCH}_{3}\right), 7.66(\mathrm{~d}, J=4.2 \mathrm{~Hz}, 1 \mathrm{H},=\mathrm{CH}-\mathrm{CO}), 7.98(\mathrm{~d}, J=8.8 \mathrm{~Hz}, 2 \mathrm{H}$, $\mathrm{ArH}), 8.13$ (d, $J=15.6 \mathrm{~Hz}, 1 \mathrm{H},=\mathrm{CH}-\mathrm{Ar})$. EI MS: $m / z$ (\%) $497\left(\mathrm{M}^{+}, 5\right), 256$ (49), 204 (79), 177 (15), 172 (25), 144 (100), 131 (30), 114 (52), 102 (33), 101 (40). Anal. Calcd for $\mathrm{C}_{27} \mathrm{H}_{22} \mathrm{Cl}_{2} \mathrm{O}_{5}$ : $\mathrm{C}$, 65.20; H, 4.46. Found: C, 65.43; H, 4.63.

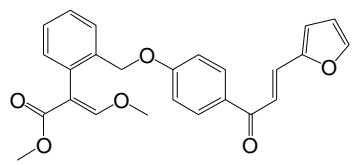

3o: Yield, 82\%. mp, 59-61 $\square .{ }^{1} \mathrm{H}$ NMR (400 MHz, $\mathrm{CDCl}_{3}$ ): $\delta: 3.71$ (s, 3H, $\mathrm{COOCH}_{3}$ ), 3.83 (s, 3H, $\left.\mathrm{OCH}_{3}\right), 5.04\left(\mathrm{~s}, 2 \mathrm{H}, \mathrm{CH}_{2}\right), 6.50-6.51(\mathrm{~m}, 1 \mathrm{H}, \mathrm{ArH}), 6.68(\mathrm{~d}, J=3.2 \mathrm{~Hz}, 1 \mathrm{H}, \mathrm{ArH}), 6.99$ (d, $J=$ $8.8 \mathrm{~Hz}, 2 \mathrm{H}, \mathrm{ArH}), 7.20-7.39$ (m, 4H, ArH), 7.52-7.59 (m, 3H, ArH, =CH-CO, =CH-Ar), 7.60 (s, $\left.1 \mathrm{H},=\mathrm{CH}-\mathrm{OCH}_{3}\right), 8.01(\mathrm{~d}, J=7.6 \mathrm{~Hz}, 2 \mathrm{H}, \mathrm{ArH})$. EI MS: $m / z(\%) 418\left(\mathrm{M}^{+}, 11\right), 204(32), 146(18)$, 144 (100), 130 (22), 120 (17), 114 (19), 102 (17), 101 (11). Anal. Calcd for $\mathrm{C}_{25} \mathrm{H}_{22} \mathrm{O}_{6}$ : C, 71.76 ; H, 5.30. Found: C, 71.69; H, 5.53.

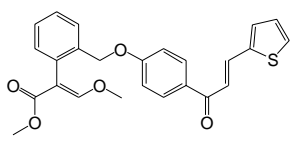

3p: Yield, 76\%. mp, 142-144 $\square .{ }^{1} \mathrm{H}$ NMR (400 MHz, $\mathrm{CDCl}_{3}$ ): $\delta: 3.72$ (s, 3H, $\mathrm{COOCH}_{3}$ ), 3.83 (s, $\left.3 \mathrm{H}, \mathrm{OCH}_{3}\right), 5.05\left(\mathrm{~s}, 2 \mathrm{H}, \mathrm{CH}_{2}\right), 6.97(\mathrm{~d}, J=8.4 \mathrm{~Hz}, 2 \mathrm{H}, \mathrm{ArH}), 7.08-7.21(\mathrm{~m}, 2 \mathrm{H}, \mathrm{ArH}), 7.30-7.50$ $(\mathrm{m}, 6 \mathrm{H}, \mathrm{ArH},=\mathrm{CH}-\mathrm{CO}), 7.60\left(\mathrm{~s}, 1 \mathrm{H},=\mathrm{CH}-\mathrm{OCH}_{3}\right), 7.92(\mathrm{~d}, J=15.6 \mathrm{~Hz}, 1 \mathrm{H},=\mathrm{CH}-\mathrm{Ar}), 7.99(\mathrm{~d}, J$ $=8.8 \mathrm{~Hz}, 2 \mathrm{H}, \mathrm{ArH})$. EI MS: $m / z(\%) 435\left([\mathrm{M}+1]^{+}, 8\right), 434\left(\mathrm{M}^{+}, 21\right), 204$ (88), $200(16), 176(15)$, 145 (62), 144 (100), 130 (43), 128 (25), 114 (47), 102 (60), 101 (25). Anal. Calcd for $\mathrm{C}_{25} \mathrm{H}_{22} \mathrm{O}_{5} \mathrm{~S}$ : C, 69.11; H, 5.10; S, 7.38. Found: C, 69.37; H, 5.12. S, 7.15. 


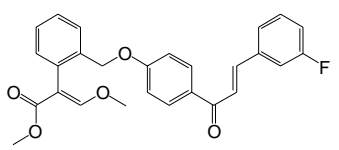

3q: Yield, 71\%. mp, 120-122 $\square .{ }^{1} \mathrm{H} \mathrm{NMR}\left(400 \mathrm{MHz}, \mathrm{CDCl}_{3}\right.$ ): $\delta: 3.72$ (s, 3H, $\mathrm{COOCH}_{3}$ ), 3.84 (s, $\left.3 \mathrm{H}, \mathrm{OCH}_{3}\right), 5.06\left(\mathrm{~s}, 2 \mathrm{H}, \mathrm{CH}_{2}\right), 6.99(\mathrm{~d}, J=6.8 \mathrm{~Hz}, 2 \mathrm{H}, \mathrm{ArH}), 7.10-7.26(\mathrm{~m}, 2 \mathrm{H}, \mathrm{ArH}), 7.31-7.39$ $(\mathrm{m}, 5 \mathrm{H}, \mathrm{ArH}), 7.49-7.53(\mathrm{~m}, 2 \mathrm{H}, \mathrm{ArH},=\mathrm{CH}-\mathrm{CO}), 7.60\left(\mathrm{~s}, 1 \mathrm{H},=\mathrm{CH}-\mathrm{OCH}_{3}\right), 7.73(\mathrm{~d}, J=15.6 \mathrm{~Hz}$, $1 \mathrm{H},=\mathrm{CH}-\mathrm{Ar}), 8.00(\mathrm{~d}, J=7.2 \mathrm{~Hz}, 2 \mathrm{H}, \mathrm{ArH})$. EI MS: $m / z(\%) 446\left(\mathrm{M}^{+}, 10\right), 204$ (41), 182 (13), 164 (23), 145 (29), 144 (100), 130 (26), 120 (15), 114 (24), 102 (26). Anal.Calcdfor $\mathrm{C}_{27} \mathrm{H}_{23} \mathrm{FO}_{5}$ : C, 72.63; H, 5.19. Found: C,72.87; H, 5.34

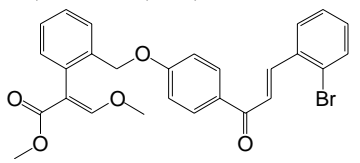

3r: Yield, 83\%. mp, 114-116 $\square .{ }^{1} \mathrm{H}$ NMR (400 MHz, $\left.\mathrm{CDCl}_{3}\right): \delta: 3.71$ (s, 3H, $\left.\mathrm{COOCH}_{3}\right), 3.84$ (s, $\left.3 \mathrm{H}, \mathrm{OCH}_{3}\right), 5.06\left(\mathrm{~s}, 2 \mathrm{H}, \mathrm{CH}_{2}\right), 6.99(\mathrm{~d}, J=8.8 \mathrm{~Hz}, 2 \mathrm{H}, \mathrm{ArH}), 7.20-7.44(\mathrm{~m}, 5 \mathrm{H}, \mathrm{ArH})$, 7.43-7.51(m, 2H, ArH), 7.60-7.64 (m, 2H, ArH, $=$ C$\left.\underline{H}-\mathrm{OCH}_{3}\right), 7.72(\mathrm{~d}, J=6.4 \mathrm{~Hz}, 1 \mathrm{H},=\mathrm{CH}-\mathrm{CO})$, $8.00(\mathrm{~d}, J=8.8 \mathrm{~Hz}, 2 \mathrm{H}, \mathrm{ArH}), 8.12(\mathrm{~d}, J=15.6 \mathrm{~Hz}, 1 \mathrm{H},=\mathrm{CH}-\mathrm{Ar})$. EI MS: $m / z(\%) 508\left([\mathrm{M}+1]^{+}, 5\right)$, 507 (M+, 4), 427 (13), 224 (14), 222 (72), 204 (99), 193 (47), 176 (59), 173 (86), 164 (54), 144 (100), 130 (68), 128 (33), 120 (47), 117 (32), 114 (64), 102 (87). Anal. Calcd for $\mathrm{C}_{27} \mathrm{H}_{23} \mathrm{BrO}_{5}$ : C, 63.92; H, 4.57. Found: C, 64.21; H, 4.67.

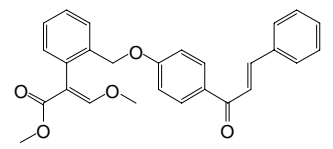

3s: Yield, 80\%. mp, 104-106 $\square .{ }^{1} \mathrm{H}$ NMR (400 MHz, $\left.\mathrm{CDCl}_{3}\right): \delta: 3.72\left(3 \mathrm{H}, \mathrm{s}, \mathrm{COOCH}_{3}\right), 3.84(\mathrm{~s}$, $\left.3 \mathrm{H}, \mathrm{OCH}_{3}\right), 5.06\left(\mathrm{~s}, 2 \mathrm{H}, \mathrm{CH}_{2}\right), 6.99(\mathrm{~d}, J=4.4 \mathrm{~Hz}, 2 \mathrm{H}, \mathrm{ArH}), 7.19-7.25(\mathrm{~m}, 2 \mathrm{H}, \mathrm{ArH}), 7.31-7.53$ $(\mathrm{m}, 7 \mathrm{H}, \mathrm{ArH},=\mathrm{CH}-\mathrm{CO}), 7.60-7.75\left(\mathrm{~m}, 3 \mathrm{H}, \mathrm{ArH},=\mathrm{CH}-\mathrm{OCH}_{3},=\mathrm{CH}-\mathrm{Ar}\right), 8.00(\mathrm{~d}, J=8.8 \mathrm{~Hz}, 2 \mathrm{H}$, ArH). EI MS: $m / z(\%) 429\left([\mathrm{M}+1]^{+}, 7\right), 428\left(\mathrm{M}^{+}, 18\right), 204$ (100), 167 (18), 165 (31), 152 (21), 146 (60), 145 (99), 131 (59), 114 (41). Anal. Calcd for $\mathrm{C}_{27} \mathrm{H}_{24} \mathrm{O}_{5}$ : C, 75.68; H, 5.65. Found: C, $75.81 ; \mathrm{H}, 5.42$.

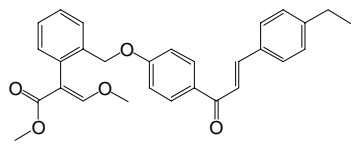

3t: Yield, 79\%. mp, 181-183 $\square .{ }^{1} \mathrm{H}$ NMR (400 MHz, $\mathrm{CDCl}_{3}$ ): $\delta: 1.25$ (t, $J=7.6 \mathrm{~Hz}, 3 \mathrm{H}, \mathrm{CH}_{3}$ ), $2.68\left(\mathrm{dd}, J_{1}=6.8 \mathrm{~Hz}, J_{2}=12.0 \mathrm{~Hz}, 2 \mathrm{H}, \mathrm{CH}_{2} \mathrm{CH}_{3}\right), 3.71\left(\mathrm{~s}, 3 \mathrm{H}, \mathrm{COOCH}_{3}\right), 3.84\left(\mathrm{~s}, 3 \mathrm{H}, \mathrm{OCH}_{3}\right), 5.06$ (s, $\left.2 \mathrm{H}, \mathrm{CH}_{2}\right), 6.96(\mathrm{~d}, J=8.8 \mathrm{~Hz}, 2 \mathrm{H}, \mathrm{ArH}), 7.23-7.36(\mathrm{~m}, 5 \mathrm{H}, \mathrm{ArH}), 7.47-7.57(\mathrm{~m}, 4 \mathrm{H}, \mathrm{ArH}$, $=\mathrm{CH}-\mathrm{CO}), 7.60\left(\mathrm{~s}, 1 \mathrm{H},=\mathrm{CH}-\mathrm{OCH}_{3}\right), 7.76(\mathrm{~d}, J=15.6 \mathrm{~Hz}, 1 \mathrm{H},=\mathrm{CH}-\mathrm{Ar}), 8.00(\mathrm{~d}, J=8.8 \mathrm{~Hz}, 2 \mathrm{H}$, ArH). EI MS: $m / z(\%) 457\left([\mathrm{M}+1]^{+}, 5\right), 456\left(\mathrm{M}^{+}, 13\right), 251$ (21), 223 (33), 204 (97), 176 (41), 172 (48), 164 (25), 144 (100), 130 (64), 129 (26), 114 (58), 102 (33), 101 (28). Anal. Calcd for $\mathrm{C}_{29} \mathrm{H}_{28} \mathrm{O}_{5}$ : C, 76.30; H, 6.18. Found: C,76.59; H, 6.26.

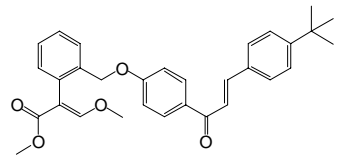

3u: Yield, 81\%. mp, 139-141 $\square .{ }^{1} \mathrm{H}$ NMR (400 MHz, $\left.\mathrm{CDCl}_{3}\right): \delta: 1.34$ (s, 9H, $\left.\mathrm{CH}_{3}\right), \quad 3.71(\mathrm{~s}, 3 \mathrm{H}$, $\left.\mathrm{COOCH}_{3}\right), 3.84\left(\mathrm{~s}, 3 \mathrm{H}, \mathrm{OCH}_{3}\right), 5.06\left(\mathrm{~s}, 2 \mathrm{H}, \mathrm{CH}_{2}\right), 6.98(\mathrm{~d}, J=9.2 \mathrm{~Hz}, 2 \mathrm{H}, \mathrm{ArH}), 7.18-7.25(\mathrm{~m}$, $1 \mathrm{H}, \mathrm{ArH}), 7.32-7.60\left(\mathrm{~m}, 9 \mathrm{H}, \mathrm{ArH},=\mathrm{CH}-\mathrm{CO},=\mathrm{C}^{\mathrm{H}}-\mathrm{OCH}_{3}\right), 7.76(\mathrm{~d}, J=16.0 \mathrm{~Hz}, 1 \mathrm{H},=\mathrm{CH}-\mathrm{Ar})$, 
$8.00(\mathrm{~d}, J=8.8 \mathrm{~Hz}, 2 \mathrm{H}, \mathrm{ArH})$. EI MS: $m / z(\%) 484\left(\mathrm{M}^{+}, 3\right), 205$ (28), 204 (36), 172 (13), 145 (100), 144 (94), 130 (28), 115 (24), 102 (28), 101 (28). Anal. Calcd for $\mathrm{C}_{31} \mathrm{H}_{32} \mathrm{O}_{5}: \mathrm{C}, 76.84 ; \mathrm{H}$, 6.66. Found: C,76.98; H, 6.96.

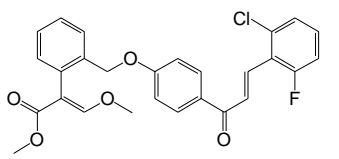

3v: Yield, 84\%. mp, 62-64 $\square .{ }^{1} \mathrm{H}$ NMR (400 MHz, $\left.\mathrm{CDCl}_{3}\right): \delta: 3.71\left(\mathrm{~s}, 3 \mathrm{H}, \mathrm{COOCH}_{3}\right), 3.84(\mathrm{~s}, 3 \mathrm{H}$, $\left.\mathrm{OCH}_{3}\right), 5.06\left(\mathrm{~s}, 2 \mathrm{H}, \mathrm{CH}_{2}\right), 6.98(\mathrm{~d}, J=8.8 \mathrm{~Hz}, 2 \mathrm{H}, \mathrm{ArH}), 7.07-7.35(\mathrm{~m}, 7 \mathrm{H}, \mathrm{ArH}), 7.51(\mathrm{~d}, J=$ $8.8 \mathrm{~Hz}, 1 \mathrm{H},=\mathrm{CH}-\mathrm{CO}), 7.60\left(\mathrm{~s}, 1 \mathrm{H},=\mathrm{CH}-\mathrm{OCH}_{3}\right), 7.78(\mathrm{~d}, J=16.4 \mathrm{~Hz}, 1 \mathrm{H},=\mathrm{CH}-\mathrm{Ar}), 8.00(\mathrm{~d}, J=$ 9.2Hz, 2H, ArH). EI MS: $m / z(\%) 481\left([\mathrm{M}+1]^{+}, 5\right), 480\left(\mathrm{M}^{+}, 8\right), 275$ (17), 241 (43), 204 (90), 172 (33), 144 (100), 130 (31), 114 (45), 102 (32), 101 (27). Anal. Calcd for $\mathrm{C}_{27} \mathrm{H}_{22} \mathrm{ClFO}_{5}$ : C, 67.43; H, 4.61. Found: C, 67.42; H, 4.91. 